\title{
An Enhanced Spectroscopic Census of the Orion Nebula Cluster
}

\author{
Lynne A. Hillenbrand ${ }^{1}$ \\ lah@astro.caltech.edu \\ Aaron S. Hoffer ${ }^{1,2}$ \\ hofferaa@msu.edu \\ and \\ Gregory J. Herczeg ${ }^{1,3}$ \\ gherczeg1@gmail.com
}

\begin{abstract}
We report new spectral types or spectral classification constraints for over 600 stars in the Orion Nebula Cluster (ONC) based on medium resolution $(R \approx 1500-2000)$ red optical spectra acquired using the Palomar 200" and Kitt Peak 3.5m telescopes. Spectral types were initially estimated for F, G, and early K stars from atomic line indices while for late $\mathrm{K}$ and $\mathrm{M}$ stars, constituting the majority of our sample, indices involving $\mathrm{TiO}$ and $\mathrm{VO}$ bands were used. To ensure proper classification, particularly for reddened, veiled, or nebula-contaminated stars, all spectra were then visually examined for type verification or refinement. We provide an updated spectral type table that supersedes Hillenbrand (1997), increasing the percentage of optically visible ONC stars with spectral type information from $68 \%$ to $90 \%$. However, for many objects, repeated observations have failed to yield spectral types primarily due to the challenges of adequate sky subtraction against a bright and spatially variable nebular background. The scatter between our new and our previously determined spectral types is approximately 2 spectral sub-classes. We also compare our grating spectroscopy results with classification based on narrow-band TiO filter photometry from da Rio et al. (2010, 2012), finding similar scatter. While the challenges of working in the ONC may explain much of the spread, we highlight several stars showing significant and unexplained bona fide spectral variations in observations taken several years apart; these and similar cases could be due to a combination of accretion and extinction changes. Finally, nearly $20 \%$ of ONC stars exhibit obvious Ca II triplet emission indicative of strong accretion.
\end{abstract}

\footnotetext{
${ }^{1}$ Department of Astronomy, California Institute of Technology, Pasadena, CA 91125

${ }^{2}$ Current affiliation: Department of Physics and Astronomy, Michigan State University, East Lansing, 48823

${ }^{3}$ Current affiliation: Kavli Institute for Astronomy and Astrophysics, Peking University; Yi He Yuan Lu 5, Hai Dian Qu; Beijing 100871, P. R. China
} 


\section{Introduction}

\subsection{The Orion Nebula Cluster}

Located at less than one-half kpc from the Sun and at moderate galactic latitude $(b \approx-20)$, the greater Orion region is the nearest example of recent and ongoing massive star formation; see Bally (2008) for an overview. A subclustered OB association extends over many tens of pc and contains stars up to a few tens of Myr old. To the southeast, the Orion A and B molecular cloud complex harbors stellar nurseries each only a fraction of a pc to a few pc in size, and less than one Myr old. The Orion composite is a revered touch stone for our developing understanding of star and planet formation processes on this range of spatial and temporal scales. The identification and detailed study of the young stellar population across the Orion complex is our best opportunity for probing in three dimensions a resolved star formation history.

Part of the so-called "integral shaped filament" of molecular gas (Bally et al. 1987), the northern part of the Orion A cloud has up to 100 magnitudes of total visual extinction through its densest regions. The Orion Nebula, also known as M42 or NGC 1976, betrays a rich young stellar cluster, the Orion Nebula Cluster (ONC), the center of which contains the massive Trapezium stars 1. The current three-dimensional model of the Orion Nebula indicates a thin shell of ionized gas (Wen \& O'Dell 1995) with the main ionizing source region $\theta^{1}$ Ori C. Another $\sim 20$ stars A0 or earlier are within the HII region as is a sizable population (many thousand members) of later spectral type T Tauri stars. These lower mass stars are still pre-main sequence and can be used to trace the star formation history as well as measure the initial mass function (IMF), which in the case of young, unevolved and co-located stars, is equivalent to the present day mass function.

In contrast to the sparsely distributed and older OB association, the ONC is relatively compact and more recently formed. Its projection on molecular cloud material significantly reduces contamination by background stars. However, the high and spatially variable extinction (e.g. Scandariato et al. 2011) renders fewer than $50 \%$ of the $\sim 3500$ stars within this region having $K<14 \mathrm{mag}$ also optically bright, $I<17.5 \mathrm{mag}$. Nevertheless, the bulk of the optically visible ONC members are extincted by 2 magnitudes or less, with derived extinction values for fainter stars ranging up to 8 magnitudes. The deepest optical surveys as well as most near-infrared surveys measure more heavily extincted members, but are more likely to suffer contamination at the faint end - both from intrinsically bright but highly reddened background giants seen through the cloud, and from intrinsically faint foreground and unreddened but late type field dwarfs.

Cluster membership can be determined kinematically in the ONC. Using astrometric techniques, Jones \& Walker (1988) identified 891 probable members from 997 measured proper motions (measured among 1052 optically visible stars). Radial velocities (e.g. Tobin et al. 2009) have also

\footnotetext{
${ }^{1}$ Traditionally, the Trapezium Cluster is the densest part of the ONC within about $2^{\prime}$ (corresponding to $\sim 0.3 \mathrm{pc}$ ), while the larger ONC extends to about $20^{\prime}(<3 \mathrm{pc})$
} 
been used to establish cluster membership. High precision three-dimensional space motion data from these and other ongoing investigations can be combined with precise cluster distance estimates (Jeffries 2007; Kraus et al. 2007; Hirota et al. 2007; Sandstrom et al. 2007; Menten et al. 2007), to study the cluster structure and dynamics and test theories for formation and evolution of dense clusters in molecular clouds. Additional observation and analysis work in this area is needed.

The ONC contains a broadly populated stellar mass function extending from a single massive (late-O type) star all the way down to low-mass stars (having late M types) and into the sub-stellar mass regime of brown dwarfs (likely L and T type), as well as planetary mass objects remaining to be spectrally typed and identified as such. With a mean stellar age for the optically visible stars of less than 2 Myr (Herbig \& Terndrup 1986; Hillenbrand 1997; da Rio et al. 2010; Reggiani et al. 2011), the ONC is not only one of the largest but also one of the densest $\left(\sim 10^{4} \mathrm{stars} \mathrm{pc}^{-3}\right.$ in the inner cluster; McCaughrean \& Stauffer (1994); Hillenbrand \& Hartmann (1998)) nearby star forming regions. The ONC population was used to demonstrate definitively that it is possible to form both high and low mass stars as well as brown dwarfs in the same $<0.1$ pc region. From the lower mass stars, the estimated current star formation rate is $\sim 10^{-4} M_{\odot}$ per year (Hillenbrand 1997, hereafter H97).

The H97 study of the ONC employed optical spectroscopy and photometry to individually de-redden and locate over 900 stars on the H-R diagram, enabling estimation of their masses and ages via comparison to pre-main sequence evolutionary tracks. Work by Luhman et al. (2000), Slesnick et al. (2004), Riddick et al. (2007b) and Weights et al. (2009) increased the spectroscopically studied sample, employing optical and near-infrared spectral classification techniques. da Rio et al. (2010) and da Rio et al. (2012) estimated spectral types for M-type stars from narrow band photometry, as discussed in more detail below. Additional studies such as those of Hillenbrand \& Carpenter (2000), Muench et al. (2002), Lucas et al. (2005), and Andersen et al. (2011) used photometric techniques rather than spectroscopic, and arrived at IMF estimates that are useful in a statistical sense but do not determine masses and ages for individual stars. The general consensus from the above studies is that the ONC IMF rises from the highest masses to sub-solar masses, realizes a flattening in the $0.5-0.6 M_{\odot}$ regime, and peaks in the $0.2-0.3 M_{\odot}$ regime before turning over throughout the sub-stellar (brown dwarf) mass range. There is some discrepancy as to the exact form of the IMF below the peak and turnover in this cluster (and in many other young clusters). Increased spectroscopic samples as well as improved understanding of contraction models at young ages will be required for progress on the IMF in the low mass stellar and sub-stellar domain.

Recent reviews of the ONC region are those by Muench et al. (2008) on the stellar population and historical distance measurements, and by O'Dell et al. (2008) on the local ONC interstellar medium including circumstellar structures. The ONC stellar population has been the sample of choice for investigations of various stellar and circumstellar phenomena, in addition to the IMF studies described above, in part because it is the nearest and youngest example of an entire stellar/sub-stellar mass spectrum. Recent studies of circumstellar dust and gas include those of Megeath et al. (2012), Mann \& Williams (2009) and Ricci et al. (2008), of disk accretion include 
Manara et al. (2012) and Sicilia-Aguilar et al. (2005), and of variability-disk connections include Morales-Calderón et al. (2012). Recent studies on stellar properties include topics such as rotation and disk-rotation connections (Rhode et al. 2001; Carpenter et al. 2001; Herbst et al. 2002;

Herbst \& Mundt 2005; Rebull et al. 2006; Cieza \& Baliber 2007), lithium depletion (Palla et al. 2007), coronal activity/flaring (Feigelson et al. 2002; Getman et al. 2008; Prisinzano et al. 2008), stellar abundances (Cunha \& Smith 2005), magnetic fields (Petit et al. 2008), and multiplicity (e.g. Simon et al. 1999; Reipurth et al. 2007; Kohler et al. 2006).

\subsection{Motivation for Further Spectroscopic Study}

There is still great interest in improving our knowledge of stellar and circumstellar properties within the ONC. As described above, the H97 paper and its accompanying database have been used in subsequent years by many other investigators studying e.g. the stellar/sub-stellar IMF, the age distribution and star formation history, stellar/sub-stellar angular momentum evolution, circumstellar disks and disk evolution, accretion, magnetic activity, lithium, as well as other properties of young stars in the ONC. Recent examples of major surveys of the ONC with forefront facilities that reach unprecedented depth across the electromagnetic spectrum, include those from X-ray (with Chandra; PI E. Feigelson) to optical (with Hubble; PI M. Robberto) to mid-infrared (with Spitzer; Megeath et al. (2012)) and finally to millimeter (with the SMA and CARMA interferometers). Synoptic photometric monitoring studies by various groups at optical and infrared wavelengths (e.g. Morales-Calderón et al. 2011) continue.

Nearly all of these previous analyses and new surveys are limited in their analysis phases, however, due to lack of completeness of the ONC spectroscopic survey. Critically important is that due to the wide range in line-of-sight extinction values to individual stars, spectra are necessary for de-reddening and thus derivation of extinction estimates, and for subsequent placement on the HR diagram from which stellar quantities such as stellar luminosity, stellar radius, and (further guided by theory) stellar age and mass, angular momentum, etc.

The optical color-magnitude diagram for the ONC suggests that the H97 optical photometric database is sensitive down to the hydrogen burning limit for stars less obscured than 2 magnitudes of visual extinction and younger than $1 \mathrm{Myr}$ (e.g. da Rio et al. 2010, H97). Infrared surveys extend well into the brown dwarf regime with the stellar-substellar boundary identified at spectral type M6.5 given the age of the ONC. While several thousand young objects are known, only about $63 \%$ of the 1500 stars with $I<17$ mag had spectral types following the H97 study (and only about 5\% before). Considering both optical and infrared-only sources in the region, less than $25 \%$ of the total number of known sources have had their spectral types determined. While infrared spectroscopy will be required in order to obtain a complete spectroscopic census of the ONC stellar population, optical spectral types can be obtained for several hundred more objects than have been published thus far. 
The present work aims to enhance the H97 optical spectroscopic census. By completing our optical study of the stellar population, it will be possible for other authors to revisit interests in many of the above goals.

\subsection{Optical Spectral Typing and Challenges in the ONC}

Spectral type is a key characteristic of any star, and spectral typing is a typical first step in estimating the stellar effective temperature. This can lead, in combination with other data or assumptions, to derivation of other stellar quantities. The optical wavelength range is important for spectral typing because it contains a rich set of molecular and atomic absorption features that are temperature dependent. $\mathrm{M}$ type stars, which dominate the mass function, are characterized by metallic oxide $\mathrm{TiO}$ and $\mathrm{VO}$ bands. These molecular features diminish at hotter temperatures with mid-K and earlier stars classified using atomic line strengths and ratios involving e.g. Ca I and Fe I. Reliable surface gravity signatures include the shapes of some of the molecular bands as well as the strength of the Na I doublet $(\lambda \lambda 8183,8194 \AA)$ for spectral types later than M2. For earlier type stars, most reliable surface gravity indicators lie blueward of our spectral coverage.

Relevant to the ONC, spectral types can be determined largely independent of reddening and nebular emission when these effects are modest, though in extreme cases they do bias or even hinder the analysis. For late type spectra, the ratios of the depths of metal oxide wavebands are not significantly affected by low reddening and the possibility of reddening can be taken into account when assigning types. Nebular emission, specfically from low Balmer (e.g. H $\alpha$ ) and high Paschen lines as well as the Paschen continuum can also affect the spectral typing process or even prevent it in extreme cases, especially for faint objects or if background subtraction (a combination of terrestrial sky and ONC nebular backgrounds) is ineffective. For late type stars the TiO and VO bands are generally strong enough such that the effects of minor nebular emission can be overcome. For earlier spectral type stars, however, strong nebular contamination is more problematic for spectral typing.

A final effect is that of non-nebular continuum and emission lines that are associated in young

stars with disk-to-star accretion processes. Spectral typing can be influenced by non-photospheric contributions to emergent spectrum and in extreme cases the spectrum is accretion-dominated.

\section{New Optical Spectroscopic Observations}

We obtained low-resolution optical spectra in 15 configurations with the HYDRA multi-object spectrograph on the 3.5m WIYN telescope at Kitt Peak during two runs in 2006-2007. We also used the (now decomissioned) Norris multi-object spectrograph on the $5 \mathrm{~m}$ Hale Telescope at Palomar in 1999 to obtain 1 configuration on the ONC at the very end of a night allocated to another program. HYDRA consists of a $1024 \times 2048$ CCD fed with 99 fibers, each with a $2^{\prime \prime}$ aperture (Barden 
\& Armandroff 1995). The field size is $60^{\prime}$ in diameter. We used the $316 \mathrm{l} / \mathrm{mm}$ grating blazed at $7500 \AA$ setting of the bench spectrograph and the GG-495 filter to obtain spectra from 5000-10000 $\AA$ at $R \sim 1500$. Norris consisted of a 2048x2048 CCD illuminated with 176 fibers, each with a 1." 5 aperture (Hamilton et al. 1993). The field size was $20^{\prime}$ in diameter. Our Norris observations were obtained with the $600 \mathrm{l} / \mathrm{mm}$ grating blazed at $5000 \AA$ to produce $54 \AA / \mathrm{mm}$ dispersion $(1.3$ $\AA /$ pixel). The spectral range is $6100-8750 \AA$ at $R \sim 2000$.

Table 1 contains the observation log. Fiber assignments were prioritized based on ancillary information such as existence of a published rotation period, exhibition of large amplitude variability, and proper motion membership probability; however, our data set is sizable and deep enough that these original prioritization criteria are not a significant bias. Both instruments were configured to maximize the total number of quality science targets and minimize the number of repeated spectra. However there are many cases of multiple observations of the same object. This is useful mitigation against sky subtraction challenges and also provides independent assessment of spectral types.

In each fiber configuration, between 30-120 fibers were assigned to a stellar position and between 13-54 fibers were assigned to sky positions. The set of observations for each configuration includes a long exposure on-target, a shorter sky exposure offset $6^{\prime \prime}-10^{\prime \prime}$ from the target position, and a set of dome flats and comparison lamps exposures. The nearby sky exposure permits accurate correction for nebulosity, which is spatially variable and hence not accurately represented in averaged spectra of all in-field sky fibers. During our Norris observations we obtained three on-target and one offset sky integration. During our HYDRA observations the number of on-target and sky position integrations varied. Total exposure times are listed in Table 1.

\section{Raw Data Processing and Spectral Extraction}

The observations were reduced using custom routines written in $I D L$. The bias level was corrected in each image using the CCD overscan region and the flatfield correction appropriate to each fiber was applied. The trace of each fiber on the detector was determined using the dome flat with the absolute position of each fiber independently derived for each individual exposure. Cosmic rays were corrected by identifying deviations from the illumination profile across each fiber on the detector. Scattered light was accounted for by fitting a spline to pixels between fiber positions. The counts in each fiber were re-sampled onto a sub-pixel scale across the dispersion axis to ensure that the extraction window remained constant for each separate on-target, sky, and flat exposure within a given configuration. Background counts were subtracted for each fiber based on the counts between fiber positions.

The spectrum from each fiber was extracted using a window approximately 1.5 times the average fiber FWHM on the detector, or 5 pixels in the HYDRA spectra and 6 pixels in the Norris spectra. The 2nd-order wavelength solution (a FeAr lamp for the Norris observations and a $\mathrm{CuAr}$ lamp for the HYDRA observations) was calculated independently for each fiber. We obtained final 
spectra by summing the counts extracted for a given fiber from each on-target integration and correcting for sky and nebular emission by subtracting the counts extracted for the same fiber in the sky exposure, scaled to the difference in observing times. In several configurations the sky emission was scaled by an additional 10-20\% to account for changes in the sky transmission. A master sky spectrum was created for each fiber configuration by combining the spectra obtained from all fibers assigned to the sky, accounting for fiber-to-fiber sensitivity differences. Changes in the sky emission could be accounted for by comparing the master sky spectrum to that obtained in individual on-target and sky integrations.

Figure 1 shows the distribution of signal-to-noise for the data set, separated by fiber configuration. Despite the significant variation in data quality among the configurations (due to weather; see Table 1), overlays of spectra of the same object repeated in different fiber configurations show remarkable agreement in the continuum slope. That stated, some configurations are generally poor (e.g. M1) while others (such as f2c and f1d) are quite good and these quality factors are taken into account when assigning final spectral types.

In total we obtained 936 spectra, of which 707 were unique objects and 229 were duplicates of the same star obtained in more than one fiber configuration. In some cases the duplication was intentional due to poor signal-to-noise in the first obtained spectrum. In other cases, fibers that could not be assigned to previously unobserved targets were assigned to stars with existing spectra rather than random sky positions. When we have more than one spectrum, we typically have 2 , but in a few cases 3-6 different spectra of the same star were obtained.

\section{Analysis}

Stellar spectral types, spectral type ranges, or spectral type limits are derivable for 619 unique stars based on photospheric absorption lines. An additional 88 unique stars were observed, but no spectral type could be ascertained. The unclassifiable spectra were either low signal-to-noise ratio (the minority) or dominated by contamination from bright nebular continuum and/or strong line emission (the majority) that could not be removed using our sky subtraction techniques. A small fraction, however, are extreme accreting objects in which the continuum excess and line emission is similar to nebular contamination, but originates in the circumstellar environment. In cases of strong Ca II triplet emission the two possibilities can be distinguished, but in neither scenario can an underlying spectral type be determined. Consistent with convention, the objects lacking absorption features but having strong Ca II triplet emission are noted as "cont+emis".

Figure 2 shows distributions of I-band magnitudes taken from H97 for all stars analyzed in that paper, as well as for the subset that had spectral types at that time, and also for those that are spectral typed in this work. The distributions are somewhat similar but the new set of spectra is biased towards the fainter end of the magnitude distribution. In this section we discuss the spectral classification process including its sensitivity to effective temperature and surface gravity 
(Section 4.1), then our quality control procedures (Section 4.2). Our results are presented in Table 2 (Section 4.3). Finally we discuss the Ca II emitters which exhibit unambiguous evidence for disk-to-star accretion (Section 4.4).

\subsection{Spectral Classification Procedure}

To classify the spectra we follow the same procedures as are described in Section 3.3 of H97. Specifically, the classification was performed using spectral indices to measure feature depths, in the spirit of O'Connell (1973), and followed up by visual examination of the data. For each feature, 30 $\AA$ wide bandpasses were used in creating spectral strength indices from defined on-feature and offfeature wavelength ranges; average fluxes over these bands were calculated. Riddick et al. (2007a) give a thorough description of the relevant molecular and atomic features and their behavior with temperature and surface gravity, as well as the challenges and pitfalls in classifying young stars associated with star formation regions. These effects include accounting for the observational influence of low surface gravity, accretion, large and variable reddening, and nebular contamination in the classification process.

The vast majority of the stars in the optically visible ONC sample are young late $\mathrm{K}$ and $\mathrm{M}$ type stars. For their classification metal oxide bands were used: four of $\mathrm{TiO}(\lambda 6760, \lambda 7100, \lambda 7800$, $\lambda 8465)$ and two of VO: $(\lambda 7445, \lambda 7865)$. These prominent bands are mainly temperature sensitive, making them ideal for the spectral classification. Figure 3 shows a sequence of $\mathrm{K}$ and M-type spectra from the WIYN/HYDRA and P200/Norris datasets. The TiO absorption bands used to initially classify the stars are indicated. Stars earlier than mid-K do not have strong TiO features and atomic line ratios were used, such as Ca I $\lambda 6162$ vs. Na I $\lambda 5892$ for F2-K7 stars, Ca I $\lambda 6162$ vs. Mg I $\lambda 5175$, upper Paschen lines, and other Ca I and O I lines for A0-F0 stars. Spectral typing for stars earlier than late- $\mathrm{K}$ is best done blueward of our spectral range.

Classifying our red spectra quantitatively first involved two dimensional plots of spectral feature index pairs such as: $\mathrm{TiO} \lambda 6760$ vs $\mathrm{TiO} \lambda 7100$, $\mathrm{TiO} \lambda 7100$ vs $\mathrm{TiO} \lambda 7800$, and $\mathrm{VO} \lambda 7445$ vs $\mathrm{VO}$ $\lambda 7865$. These diagrams were used to initially bin the late type ONC stars by finding the spectral type along the standard star sequence that is closest to the position of the target star. Second, cubic equations were used to fit the standard sequence for each $\mathrm{TiO}$ band. The standard stars

included those at medium spectral resolution from Allen \& Strom (1995) and Kirkpatrick et al. (1991) extending up to M6 (near the brown dwarf limit) and Kirkpatrick et al. (1999) beyond this.

For the majority of stars, different feature strength ratios predicted consistent spectral types. However, using line or band indices gives only an estimate for the spectral type. Hence, after assigning preliminary spectral types by the quantitative analysis techniques described above, each spectrum was examined visually against a grid of the standard star spectra in order to verify and in some cases slightly modify the quantitative spectral types. This step was undertaken separately by two of the authors. 
Given the age estimate of the ONC, the stars were assumed to be dwarfs in the above quantitative classification methods, with surface gravity not explicitly taken into account for the initial classification. For spectral types later than about M5, however, there is a strong gravity dependence that must be considered in order to avoid classifying stars later in type than they really are. Since the $\mathrm{TiO}$ and $\mathrm{VO}$ bands acquire a distinct shape in proceeding from dwarf-like to giant-like surface gravities, particularly the red end of the TiO $\lambda 8465$ band, this could be accounted for during the visual examination part of the process. Surface gravity effects are also seen in the strength of the Na I doublet $(\lambda \lambda 8183,8194 \AA)$ at spectral types later than M2.

Indeed, being candidate pre-main sequence stars, our objects are expected to have surface gravities in between those of dwarf stars and giant stars, though closer to dwarfs. Thus following Slesnick et al. (2006a) who were themselves guided by the findings of Kirkpatrick et al. (1991), we investigated quantitatively the strength of the $\mathrm{Na}$ I $\lambda 8190$ doublet in our spectra. This feature weakens towards lower values of surface gravity (see Figure 7 of Kirkpatrick et al. (2008) for a demonstration). We show in Figure 4 the same plot utilized by Slesnick et al. (2006b) - their Figure 4 - of the gravity-sensitive $\mathrm{Na}$ I $\lambda 8190$ index vs the temperature-sensitive $\mathrm{TiO} \lambda 8465$ and $\mathrm{TiO} \lambda 7140$ indices. ONC stars later than spectral type $\sim \mathrm{M} 2$ typically exhibit the intermediate Na I $\lambda 8190$ line strengths expected from young 1-2 Myr old pre-main sequence stars. Based on this Figure, we conclude that the vast majority of the stars M3 and later in our sample can be presumed ONC members given the consistency of their surface gravities with the assumed young age of the cluster. While a few of the M dwarfs could be interlopers along the line of sight with higher surface gravity, the ONC sample at $\mathrm{I} \lesssim 17.5$ mag appears to be largely uncontaminated by foreground dwarf stars.

For those late type stars with medium to high signal-to-noise ratio and that are not severely affected by nebular contamination (which can contribute strong lines in either the on-feature or off-feature band regions) or strong accretion (which can render observed spectra too blue relative to the expected slope for the spectral type), the quantitative method is reliable and robust to visual examination. For stars with a significant amount of reddening, low signal-to-noise ratio, or both, experimentation has shown that the quantitative technique is in fact more trustworthy than ab initio examination by eye. Indeed, for most early-to-mid M type stars, the spectral types adopted were generally those assigned quantitatively. For the late $\mathrm{M}$ types, by-eye examination was more rigorous with detailed attention to surface gravity effects needed for accurate classification. At earlier types, some adjustments to the quantitative types were made for nearly all FGK stars. These were at the 1-3 sub-class level for late $\mathrm{K}$ stars but more for the earlier types for which our red region spectra include narrow features that are only weakly varying with temperature, and therefore challenging for our coarse quantitative technique. Methods employing equivalent width measurement would be more suitable for quantitative analysis than our wide bandpass methods at these earlier spectral types. As reported above, not all of the spectra were classifiable. 


\subsection{Spectral Classification Quality Control}

In order to assess the quality of the spectral typing, we can look at the sub-set of sources with more than one spectrum obtained and classified. As a reminder, we have 936 total spectra of 707 unique sources, of which 185 unique sources were observed more than once. Of these, only 89 sources have multiply derived spectral types; the remainder have only one or zero of the multiple spectra classifiable (due to either signal-to-noise or nebular contamination issues). Among the 89 stars, 78 have two classifiable spectra and 10 have three classifiable spectra. One source, H3132, had six spectra obtained of which five are classifiable. The range in the resulting, independently derived, spectral types has a median value of just one sub-class with standard deviation less than two sub-classes. Of the 89 repeated sources, 52 are typed within one sub-class and 71 within two sub-classes. The outliers with larger discrepancies tend to be G or K stars where accurate spectral typing is more difficult from red wavelength data. Notably, H3132 exhibited a range of only one subclass among the types derived from the five different spectra, which were independently classified as M5.5, M6, M5, M5.5, and M5. It is thus clear that in the presence of good data and minimal complications from astrophysical effects, the methods are robust.

However, a number of systematics are present in the Orion Nebula Cluster region that can affect the spectral types. The main one is the accuracy of the sky/nebula subtraction. Some of our spectra have a blue SED but also late-type spectral features, which indicates that some nebular contamination remains. Although this slope/spectrum discrepancy can be seen in rapid accretors as well, the Orion Nebula itself predominantly causes the effect in our data sets. Several of the multiply observed stars with large spectral type discrepancies are cases in which one spectrum has much better sky subtraction than the other. For these stars it is easy to see that the later spectral type is the more appropriate one; however, other cases may exist where we have only one spectrum and have classified a star too early, e.g. G or K type, when it is really dominated by nebular continuum that if accurately subtracted would yield a later spectral type. As noted above, the presence of a strong accretion continuum can similarly make a spectrum appear blue as well as fill in the atomic absorption lines, resulting in a spectral classification that is biased earlier than the true type and we may indeed have some of these cases. Another complication to accurate spectral typing is reddening. While we believe our methods are able to account for modest amounts of reddening, it is possible that in extreme cases there are systematic effects that enter and cause larger than average spectral type errors. A final worry is the possible presence of cool spots, similar to those seen on older non-accreting but still active young stars. These could either produce or enhance late type features on the red side of our spectral range, leading to a bias towards a later spectral type assignment than truly characterizes the underlying star. In summary, one or more of the above astrophysical effects could be present and therefore there are possible spectral type biases in both directions.

Finally, we note that for comparison purposes we also ran our spectra through the distributed "SpTclass" software developed by Hernandez (2005). We found that the combination of spectral

resolution and signal-to-noise effects, plus perhaps the lack of flux calibration in our dataset, ren- 
dered the SpTclass results suspect. Specifically, the results are somewhat degenerate for stars we classified by hand as F through K7 stars, with little useful output. Even for the standard stars in this spectral type range there was quite a lot of scatter and a bias in the F, G, and early $\mathrm{K}$ range for SpTclass to classify stars much later than their true types; in the K5-K8 range, however, there was good agreement. For the later type objects, M0-M9, we found a systematic offset in both our object classifications and tests with standard stars of approximately 2 to 2.5 spectral subtypes, now in the sense that the SpTclass results were too early compared to the actual spectral type. We believe that this comparison demonstrates the need to classify stars relative to a grid of standards taken with identical or similar equipment. Furthermore, as our spectra are not flux-calibrated having been taken with fibers rather than slits, there may be an additional explanation for the systematic effects reported above if the assumptions of the SpTclass software regarding continuum shape are being violated in our dataset. Regardless of any specific SpTclass biases, our experience suggests general caution when considering automated techniques for spectral classification, though they may be very useful when applied to data sets consistent with their development or in less challenging spectroscopy environments than the ONC.

\subsection{Spectral Type Results}

The final spectral types for our ONC sample are presented in Table 2, where 555 are from the new WIYN/Hydra data and 99 are from the new Palomar/Norris data, with 35 stars classifiable in both spectral data sets. Our newly derived spectral types are on the same system as those in H97, having been assessed by the same author. Figure 5 shows the run of apparent I-band magnitude with spectral type. The scatter at any given magnitude can be attributed to cumulative differences in foreground extinction, accretion continuum excess, and/or stellar radius. For 254 of the 619 stars, the spectral types are the first ever reported. An additional 63 stars have spectral types in other literature more recent than the H97 study and compilation, as cited in Table 2.

\subsection{CaII Emission Line Results}

The strong continuum and line emission from the Orion Nebula combined with the difficulties of sky subtraction using multi-fiber instruments in high background fields such as the ONC, means that many of the emission lines traditionally used to diagnose accretion and outflow in T Tauri stars (e.g. $\mathrm{H} \alpha$ and other Balmer lines, [NII], [SII] and other forbidden lines) can not be used to identify such objects of interest among our sample stars. However, Ca II triplet $(\lambda \lambda 8498 \AA, 8542 \AA$, and $8662 \AA)$ emission was identified by Hillenbrand et al. (1998) as being a robust accretion indicator in the ONC, even in the presence of strong nebular background since these high density Ca II lines typically are not seen in nebular spectra at low spectral resolution. With the line strengths and profile widths that are observed in young stars, Ca II triplet emission is attributed to accretion processes

rather than to chromosphere activity that would produce weaker lines (Herbig \& Soderblom 1980; 
Hamann \& Persson 1992; Soderblom et al. 1993; Azevedo et al. 2006; Kwan \& Fischer 2011). The physical conditions responsible for the emission appear to require high density, $n_{H} \sim 10^{12} \mathrm{~cm}^{-3}$ and moderate to low temperature, $T<7500 \mathrm{~K}$ (Kwan \& Fischer 2011).

Approximately $20 \%$ of our stars exhibit Ca II triplet emission, consistent with the fraction reported in the earlier Hillenbrand et al. (1998) study. Figure 6 shows some example spectra of strong, medium, and weak emitters which occur mainly among the late $\mathrm{K}$ and early $\mathrm{M}$ spectral types while Figure 7] shows a histogram of the measured Ca II $\lambda 8542$ (pseudo) equivalent widths. We measured equivalent widths using splot in IRAF for each of the Ca II triplet lines against the pseudo-continuum, as well as the nearby O I $8446 \AA$ line. Notes were made concerning the strength of the upper Paschen lines and whether the Ca II lines have significant H I contamination (plausibly from strong accretion, but more likely from incomplete nebular subtraction). Our identification of emitters has required that the Ca II line series either have the $8542 \AA$ line stronger than any $8600 \AA$ pure H I, or $8498 \AA$ stronger than $8542 \AA$. Because the local continuum is significantly depressed for M-type photospheres relative to earlier spectral types, there is an expected spectral type dependence of the measured equivalent widths for a given constant Ca II line strength. We also note that many of the stronger emitters are variable among our several spectra, at a level up to $25 \%$.

The range of equivalent width values in the ONC fully populates the range seen in other star forming regions. A linear relationship between Ca II triplet line flux and total accretion luminosity is now well-established in the literature (e.g. Muzerolle et al. 1998; Herczeg \& Hillenbrand 2008; Dahm 2008). With knowledge of $M_{*} / R_{*}$, such measurements can lead to derivation of mass accretion rates. In the absence of stellar parameters, however, and with only equivalent widths rather then line fluxes (which would require either flux-calibrated spectra or contemporaneously obtained I-band photometry to mitigate errors due to variability) we can not derive accretion rates here. We can comment though, that the previously established correlations suggest that Ca II $\lambda 8542$ equivalent widths in the range $0.5-40 \AA$ for low mass stars correspond to accretion luminosities in the range $10^{-5}$ to $0.1 L_{\odot}$ and accretion rates from $<10^{-10}$ to $10^{-6.5} M_{\odot} / \mathrm{yr}$.

We conclude that a wide range of accretion rates characterizes the young ONC population. Once stellar parameters are better established for our sample, these several hundred young stars can be further investigated for correlations between accretion properties and stellar mass and age.

\section{Comparison with Previous Efforts}

All spectral classification results that have been derived from previous spectroscopy for the H97 optical sample are presented in Table 2, along with the spectral types newly derived in this paper. The historical data is mostly from the H97 compilation, but also included are our own revisions and 
updates based on that same spectral data set 2 as well as more recent estimates of spectral types by e.g. Luhman \& Rieke (2000), Lucas et al. (2001), Rhode et al (2001), Wolff et al. (2004), Slesnick et al. (2004), K. Stassun (private communication, 2005), Riddick et al. (2007), H.C. Stempels (private communication, 2009), Weights et al. (2009), Daemgen et al. (2012), and Correia et al. (2013) as referenced. We note that for the Weights et al. (2009), Riddick et al. (2007), and Lucas et al. (2001) papers it is not straight forward to associate the rounded or truncated coordinate naming system of the authors with objects in our list of interest, especially as the positions for nominally the same objects have changed for some sources between these various studies. We have done our best to match the reported spectral types for these low precision coordinate-based names to known objects within 2", where possible based on similarity of reported apparent magnitudes.

Spectral types from the sources listed above for stars that are fainter than the compiled H97 sample appear in Table 3. Note that unlike Table 2, the astrometry reported in Table 3 has varying precision. To the best of our knowledge, Tables 2 and 3 represent the complete list of spectroscopically determined spectral types for ONC stars. Additional photometric spectral types may be found in da Rio et al. (2010).

Considering now only Table 2, of the 1576 optically visible stars cataloged in H97, about 500 lacked a spectral type or any spectral type constraint at that time, while now just under 200 lack a spectral type estimate. Several tens of stars have only wide ranges or limits for their estimated types, that is, just constraints on the latest or the earliest type that is consistent with the observed spectrum. Many of the stars lacking adequate classification are in regions of bright nebulosity such that repeated observations at optical wavelengths have failed to yield a classifiable spectrum. Others are close companions to brighter stars and likewise have not had suitable spectra obtained.

\subsection{Comparison to Hillenbrand (1997) Spectroscopic Types}

As reported above, 619 stars are typed from our Norris and HYDRA data. While the observational focus of the new data acquisition was on those stars without previously determined spectral types, in practice we placed fibers on other ONC members rather than not assigning them at all. Thus the set of stars for which spectral types are derived in this paper overlaps significantly with those in the H97 paper. This enables comparison of results obtained with different equipment but using similar analysis methods

In the vast majority of cases there is remarkable agreement between the previously and presently reported spectral types, as can be seen from examination of Table 2. This is despite the known observational challenges and the likely influence of spectrophotometric variability due

\footnotetext{
${ }^{2}$ Some of the latest type stars were reclassified either as described in Slesnick et al. (2004) or here, based on improved attention to the effects of surface gravity on temperature diagnostics; these are somewhat subtle at low signal-to-noise ratio and were not fully appreciated at the time of the H97 study.
} 
to real changes in accretion / extinction parameters. Figure 8 illustrates the direct correlation between $\mathrm{H} 97$ and this work; the distribution exhibits a root-mean-squared scatter of 2.25 spectral sub-classes. However, some large discrepancies exist as well, notably when early-K vs mid-M spectral types have been derived for the same star. We advise in these cases that the later spectral type is likely the more accurate one since, as discussed earlier, there are plausible ways to make spectral types appear much earlier than the true type in some data.

Histograms in Figure 9 show the distribution of stellar types from H97 compared to the distribution in the new spectral dataset. While the histograms are similar in terms of rising from earlier to later types with a peak around M5, there are notably fewer extremely late type stars seen here compared to what was reported in the H97 paper.

\subsection{Comparision to da Rio $(2010,2012)$ Photometric Types}

da Rio et al. (2010) derive spectral types for 217 late type stars in the ONC based on narrow band photometry, employing a novel $\mathrm{TiO}$ photometric index defined as the measured magnitude in a $200 \AA$ wide filter centered at $6200 \AA$ relative to the magnitude at that same wavelength interpolated from a linear fit to V- and I-band photometry. da Rio et al. (2012) obtained a deeper photometric dataset and used a similar technique based on a longer wavelength $7700 \AA$ narrow band filter, to derive spectral types and extinction values for 1280 objects. The TiO index (narrow band photometric) technique is in fact similar in spirit to the spectroscopic $\mathrm{TiO}$ band indices that we have employed here. The photometric spectral types from these two da Rio studies range between M2 and M6, where the technique is valid, and have estimated errors of typically 1-2 sub-classes.

In Figure 10 we compare directly the da Rio photometric spectral types with the spectroscopic spectral types quoted in Table 2; the correlation exhibits a root-mean-squared scatter of 1.75 spectral sub-classes. In total, a sample of 437 stars is available for comparison between the spectroscopic and photometric spectral types, with all 437 of those having $7700 \AA$ TiO spectral types, and 315 having $6200 \AA \mathrm{TiO}$ spectral types as well, when both photometric methods could be applied. While the scatter seems large between the photometric and spectroscopic methods (filled symbols), it appears to be no worse at these late types than between the new spectroscopy and the old spectroscopy (open symbols) probing a slightly earlier spectral type range. It should be noted that, as discussed above, the error bars in this earlier regime are larger than those suggested at the later types from either the photometric methods (1-2 sub-classes) or the spectroscopic methods (0.5 sub-classes based on repeated robust measurements of the same star but up to 2 sub-classes including systematic effects).

da Rio et al. (2012) also devised procedures to de-redden their photometry and obtain both $\mathrm{A}_{V}$ and $\mathrm{T}_{\text {eff }}$ for individual late type stars. We have mimicked their analysis by performing synthetic photometry on our spectra based on the bandpasses specified by da Rio et al. (2012). Although the spectra we have are not flux calibrated, because the TiO index on-band and off-band filter profiles 
are so close in wavelength this should have only a minor effect. The resulting color-color diagram based on our spectra (not shown) is indeed similar in appearance to the one based on narrow band photometry that is presented in da Rio et al. (2012) - their Figures 5 and 11. Specifically, there is a narrow finger of stars having no indication of $\mathrm{TiO}$ depression but a range of reddening values, and then a spread along the $\mathrm{TiO}$ index or temperature-sensitive axis, as well as along a nearly orthogonal reddening vector.

We have further followed the da Rio et al. (2012) methodology for deriving both $\mathrm{A}_{V}$ and $\mathrm{T}_{e f f}$, finding for our sample extinction values in the 0-3 magnitude range and effective temperatures in the 2700 to $3900 \mathrm{~K}$ indicated. While illustrative of the consistency between different methods for deriving spectral types and then physical parameters from them, it should be noted that the scatter between the two TiO photometric methods ( $\mathrm{TiO} 6200 \AA$ in da Rio et al. (2010) vs TiO $7700 \AA$ in da Rio et al. (2012)) is itself 0.03 in $\log T_{e f f}$, corresponding to 1.5 spectral sub-classes in the $\mathrm{M}$ star regime, and comparable to the estimated errors in the photometric spectral typing method.

We conclude for mid-to-late M-type stars, that spectral typing through the use of narrow-band photometric methods is no less accurate in the mean than spectral typing through spectroscopic methods. For early M stars, however, there is a systematic discrepancy with later types being assigned through the photometric method than appear reasonable from the spectroscopy. This could be due to nebular contamination in the spectra, the photometry, or both.

\subsection{Spectral Type Variables}

There is good correlation demonstrated overall in Figures 8 and 10, as well as general agreement over many decades in the spectral types derived by different authors for the same stars (see Table 2). However, there are some cases where large discrepancies are apparent.

We highlight the case of JW 20 as an illustration. The H97 spectral type (based on data from 1996, February) was M3.5. However, the spectrum presented here (based on data from 2006, January) is that of a K8 star, as illustrated in Figure 11. The star is only a small-amplitude optical variable (0.05 mag; Herbst et al. 2002) with all available literature and catalog near-infrared photometry consistent within $0.1 \mathrm{mag}$ and the mid-infrared variability similarly small amplitude (Morales-Calderón et al. 2011). Nevertheless, between the two spectra taken ten years apart, the deep $\mathrm{TiO}$ and VO bands decreased in strength and Ca II triplet absorption was revealed.

We can not explain this discrepancy in terms of signal-to-noise challenges. Nor can we rationalize an explanation through observational confusion since there are no other optical or near-infrared sources within 30". Data for both spectra were taken with the WIYN/HYDRA fiber positioner and there are no indications among the other objects of problems with the pointing. Although the exact position of the sky fiber location was different, the sky subtraction method employed was the same, and we can not see a way of producing these two different spectra if sky/nebula subtraction accuracy was the only variation. 
Careful examination of Table 2 reveals several other such stars with large spectral type discrepancies. We are driven to the conclusion that in at least some cases, e.g. JW 20 and JW 798, astrophysical origin must be considered plausible. In other cases there may be explanations; as examples, for JW 625, H97 3030, and likely others, there is strong nebular contamination either on-source or in the available sky positions, while for H97 3067 a nearby brighter source could contaminate an on-source fiber spectrum during poor seeing conditions. These difficulties inherent to crowded, nebular star forming regions are non-astrophysical sources of discrepant spectral types that should be considered, in addition to low signal-to-noise, as possible classification biases.

\section{Summary}

We have presented an analysis of new low resolution optical spectra that extends and updates the catalog of spectral types for optically visible stars in the ONC. For 254 of our 619 newly typed stars, we present the first published spectral classifications. Of the approximately 1600 sources in the optical photometric catalog presented by H97, 90\% now have spectral types or spectral type constraints based on low resolution optical spectroscopy, compared to just $68 \%$ at the time of that paper and $5 \%$ previous to that paper. Many of the optical stars still have only wide ranges or limits on their spectral types, and not single valued spectral types. A similar number of optically invisible stars await the application of infrared spectral typing techniqes, which will be no less challenging given the complex background of the Orion Nebula.

The newly derived and the older optical spectral type distributions appear to have similar shape. In a point-by-point comparison, spectral classification differences are typically around 2 spectral sub-classes. We have also compared our grating spectrograph methods to narrow band photometry methods for deriving spectral types, and also found that the scatter of nearly 2 spectral sub-classes is somewhat larger than the precision estimates claimed for each method. Some young stars may exhibit real spectral variability over time.

With the current set of spectral types as well as recently available high quality optical photometry (e.g. da Rio et al. 2009) plus a substantial recent revision in the accepted distance to the ONC, it is timely to revisit not only the HR diagram and its derivatives, as in da Rio et al. (2012), but also investigations of stellar activity, stellar rotation, and circumstellar disk properties in this star forming region.

We acknowledge with gratitude the expert assistance of Todd Small concerning usage of the Palomar/Norris spectrograph and its data products, as well as team HYDRA at KPNO/WIYN. Emma Hovanec helped with the measurement and tabulation of emission lines. We thank Nicola da Rio for providing material that enabled the comparison shown in Figure 10, as well as Keivan Stassun and Eric Stempels for communication of unpublished spectral type information. Some financing for the middle stages of this work was provided in the mid-2000's through the HST/Treasury pro- 
gram on the ONC (PI Massimo Robberto). This research has made use of the SIMBAD database, operated at CDS, Strasbourg, France.

Facilities: Hale (Norris), WIYN (HYDRA).

\section{REFERENCES}

Allen, L. E., \& Strom, K. M. 1995, AJ, 109, 1379

Andersen, M., Meyer, M. R., Robberto, M., Bergeron, L. E., \& Reid, N. 2011, A\&A, 534, A10

Azevedo, R., Calvet, N., Hartmann, L., et al. 2006, A\&A, 456, 225

Bally, J. 2008, Handbook of Star Forming Regions, Volume I: The Northern Sky ASP Monograph Publications, Vol. 4. Edited by Bo Reipurth, p.459

Bally, J., Stark, A. A., Wilson, R. W., \& Langer, W. D. 1987, ApJ, 312, L45

Barden, S. C., \& Armandroff, T. 1995, Proc. SPIE, 2476, 56

Carpenter, J.M., Hillenbrand, L.A., \& Skrutskie, M.F. AJ, 121, 3160

Cieza, L., \& Baliber, N. 2007, ApJ, 671, 605

Correia, S., Duchene, G., Reipurth, B., et al. 2013, arXiv:1307.2299

Cunha, K. \& Smith, V.V. 2005, ApJ,626, 426

Dahm, S. E. 2008, AJ, 136, 521

Daemgen, S., Correia, S., \& Petr-Gotzens, M. G. 2012, A\&A, 540, A46

Feigelson, E.D., Broos, P., Gaffney, J.A. III, Garmire, G., Hillenbrand, L.A., Pravdo, S.H., Townsley, L., \& Tsuboi, Y. 2002, ApJ, 574, 258

Genzel, R., et al. 1981, ApJ, 247, 1039

Getman, K.V., Feigelson, E.D., Broos, P.S., Micela, G., \& Garmire, G.P. 2008, ApJ, 688, 418

Gualandris, A., Portegies Zwart, S., \& Eggleton, P. P. 2004, MNRAS, 350, 615

Hamann, F., \& Persson, S. E. 1992, ApJS, 82, 247

Hamilton, D., Oke, J. B., Carr, M. A., Cromer, J., Harris, F. H., Cohen, J., Emery, E., \& Blakee, L. 1993, PASP, 105, 1308

Herbig, G. H., \& Soderblom, D. R. 1980, ApJ, 242, 628 
Herbig, G. H., \& Terndrup, D. M. 1986, ApJ, 307, 609

Herbst, W. Bailer-Jones, C.A., Mundt, R., Meisenheimer, K., \& Wackermann, R. 2002, å, 396, 513

Herbst, W. \& Mundt, R. 2005, ApJ, 633, 967

Herczeg, G. J., \& Hillenbrand, L. A. 2008, ApJ, 681, 594

Hillenbrand, L. A. 1997, AJ, 113, 1733

Hillenbrand, L. A., \& Hartmann, L. W. 1998, ApJ, 492, 540

Hillenbrand, L. A., Strom, S. E., Calvet, N., Merrill, K. M., Gatley, I., Makidon, R. B., Meyer, M. R., \& Skrutskie, M. F. 1998, AJ, 116, 1816

Hillenbrand, L. A., \& Carpenter, J. M. 2000, ApJ, 540, 236

Hirota, T., et al. 2007, PASJ, 59, 897

Jeffries, R. D. 2007, MNRAS, 376, 1109

Jones, B. F., \& Walker, M. F. 1988, AJ, 95, 1755

Kwan, J., \& Fischer, W. 2011, MNRAS, 411, 2383

Kirkpatrick, J. D., Henry, T. J., \& McCarthy, D. W., Jr. 1991, ApJS, 77, 417

Kirkpatrick, J. D., Allard, F., Bida, T., et al. 1999, ApJ, 519, 834

Kirkpatrick, J. D., et al. 2008, ApJ, 689, 1295

Kohler, R., Petr-Gotzens, M. G., McCaughrean, M. J., Bouvier, J., Duchne, G., Quirrenbach, A., \& Zinnecker, H., 2006, å, 458, 461

Kraus, S., Balega, Y. Y., Berger, J.-P., Hofmann, K.-H., Millan-Gabet, R., et al. 2007, A\&A, 466, 649

Lucas, P. W., Roche, P. F., Allard, F., \& Hauschildt, P. H. 2001, MNRAS, 326, 695

Lucas, P.W., Roche, P.F., \& Tamura, M. 2005, MNRAS, 361, 211

Lucas, P.W., Weights, D.J., Roche, P.F., \& Riddick, F.C. 2006, MNRAS, 373, L60

Luhman, K. L., Rieke, G. H., Young, E. T., et al. 2000, ApJ, 540, 1016

Manara, C. F., Robberto, M., Da Rio, N., et al. 2012, ApJ, 755, 154

Mann, R. K., \& Williams, J. P. 2009, ApJ, 649, 36

McCaughrean, M. J., \& Stauffer, J. R. 1994, AJ, 108, 1382 
Megeath, S. T., Gutermuth, R., Muzerolle, J., et al. 2012, AJ, 144, 192

Menten, K. M., Reid, M. J., Forbrich, J., \& Brunthaler, A. 2007, A\&A, 474, 515

Morales-Calderón, M., Stauffer, J. R., Stassun, K. G., et al. 2012, ApJ, 753, 149

Morales-Calderón, M., Stauffer, J. R., Hillenbrand, L. A., et al. 2011, ApJ, 733, 50

Muench, A. A., Lada, E. A., Lada, C. J., \& Alves, J. 2002, ApJ, 573, 366

Muench, A., Getman, K., Hillenbrand, L., \& Preibisch, T. 2008, Handbook of Star Forming Regions, Volume I: The Northern Sky ASP Monograph Publications, Vol. 4. Edited by Bo Reipurth, p.483

Muzerolle, J., Hartmann, L., \& Calvet, N. 1998, AJ, 116, 455

O'Connell, R. W. 1973, AJ, 78, 1074

O'Dell, C. R., Muench, A., Smith, N., \& Zapata, L. 2008, Handbook of Star Forming Regions, Volume I: The Northern Sky ASP Monograph Publications, Vol. 4. Edited by Bo Reipurth, p. 544

Palla, F., \& Stahler, S. W. 1999, ApJ, 525, 772

Palla, F., Randich, S., Pavlenko, Y.V., Flaccomio, E., \& Pallavicini, R., 2007, ApJ, 659, 41

Petit, V., Wade, G. A., Drissen, L., Montmerle, T. \& Alecian, E., 2008, MNRAS, 387, L23

Prisinzano, L., Micela, G., Flaccomio, E., Stauffer, J. R., Megeath, T., Rebull, L., Robberto, M., Smith, K., Feigelson, E. D., Grosso, N., \& Wolk, S., 2008, ApJ, 677, 401

Prosser, C. F., Stauffer, J. R., Hartmann, L., Soderblom, D. R., Jones, B. F., Werner, M. W., \& McCaughrean, M. J. 1994, ApJ, 421, 517

Rebull, L. M., Stauffer, J. R., Megeath, S. T., Hora, J. L., \& Hartmann, L. 2006, ApJ, 646, 297

Reggiani, M., Robberto, M., da Rio, N., Meyer, M.R., Soderblom, D.R., Ricci, L. 2011, A\&A, 534, A 83

Reipurth, B., Guimaraes, M.M., Connelley, M.S., \& Bally, J., 2007, AJ, 134, 2272

Rhode, K.L., Herbst, W., \& Mathieu, R.D., 2001, AJ, 122, 3258

Ricci, L., Robberto, M., \& Soderblom, D.R., 2008, AJ, 136, 2136

Riddick, F. C., Roche, P. F., \& Lucas, P. W. 2007, MNRAS, 381, 1067

Riddick, F. C., Roche, P. F., \& Lucas, P. W. 2007, MNRAS, 381, 1077 
da Rio, N., Robberto, M., Soderblom, D.R., Panagia, N., Hillenbrand, L.A., Palla, F., \& Stassun, K. 2009, ApJS, 183, 261.

da Rio, N., Robberto, M., Soderblom, D. R., Panagia, N., Hillenbrand, L.A., Palla, F., Stassun, K.G. 2010, ApJ, 722, 1092

da Rio, N., Robberto, M., Hillenbrand, L. A., Henning, T., \& Stassun, K. G. 2012, ApJ, 748, 14

Rodríguez-Ledesma, M. V., Mundt, R., \& Eislöffel, J. 2009, A\&A, 502, 883

Sandstrom, K. M., Peek, J. E. G., Bower, G. C., Bolatto, A. D., \& Plambeck, R. L. 2007, ApJ, 667,1161

Scandariato, G., Robberto, M., Pagano, I., \& Hillenbrand, L. A. 2011, A\&A, 533, A38

Sicilia-Aguilar, A., Hartmann, L.W., Szentgyorgyi, A.H., Fabricant, D.G., Furesz, G., Roll, J., Conroy, M.A.; Calvet, N., Tokarz, S., \& Hernandez, J. 2005, AJ, 129, 363

Simon, M., Close, L. M., \& Beck, T. L. 1999, AJ, 117, 1375

Slesnick, C. L., Hillenbrand, L. A., \& Carpenter, J. M. 2004, ApJ, 610, 1045

Slesnick, C. L., Carpenter, J. M., \& Hillenbrand, L. A. 2006a, AJ, 131, 3016

Slesnick, C. L., Carpenter, J. M., Hillenbrand, L. A., \& Mamajek, E. E. 2006b, AJ, 132, 2665

Soderblom, D. R., Stauffer, J. R., Hudon, J. D., \& Jones, B. F. 1993, ApJS, 85, 315

Tobin, J. J., Hartmann, L., Furesz, G., Mateo, M., \& Megeath, S. T. 2009, ApJ, 697, 1103

Weights, D. J., Lucas, P. W., Roche, P. F., Pinfield, D. J., \& Riddick, F., 2009, MNRAS, 392, 817

Wen, Z., \& O’Dell, C. R. 1995, ApJ, 438, 784

Wolff, S. C., Strom, S. E., \& Hillenbrand, L. A. 2004, ApJ, 601, 979 


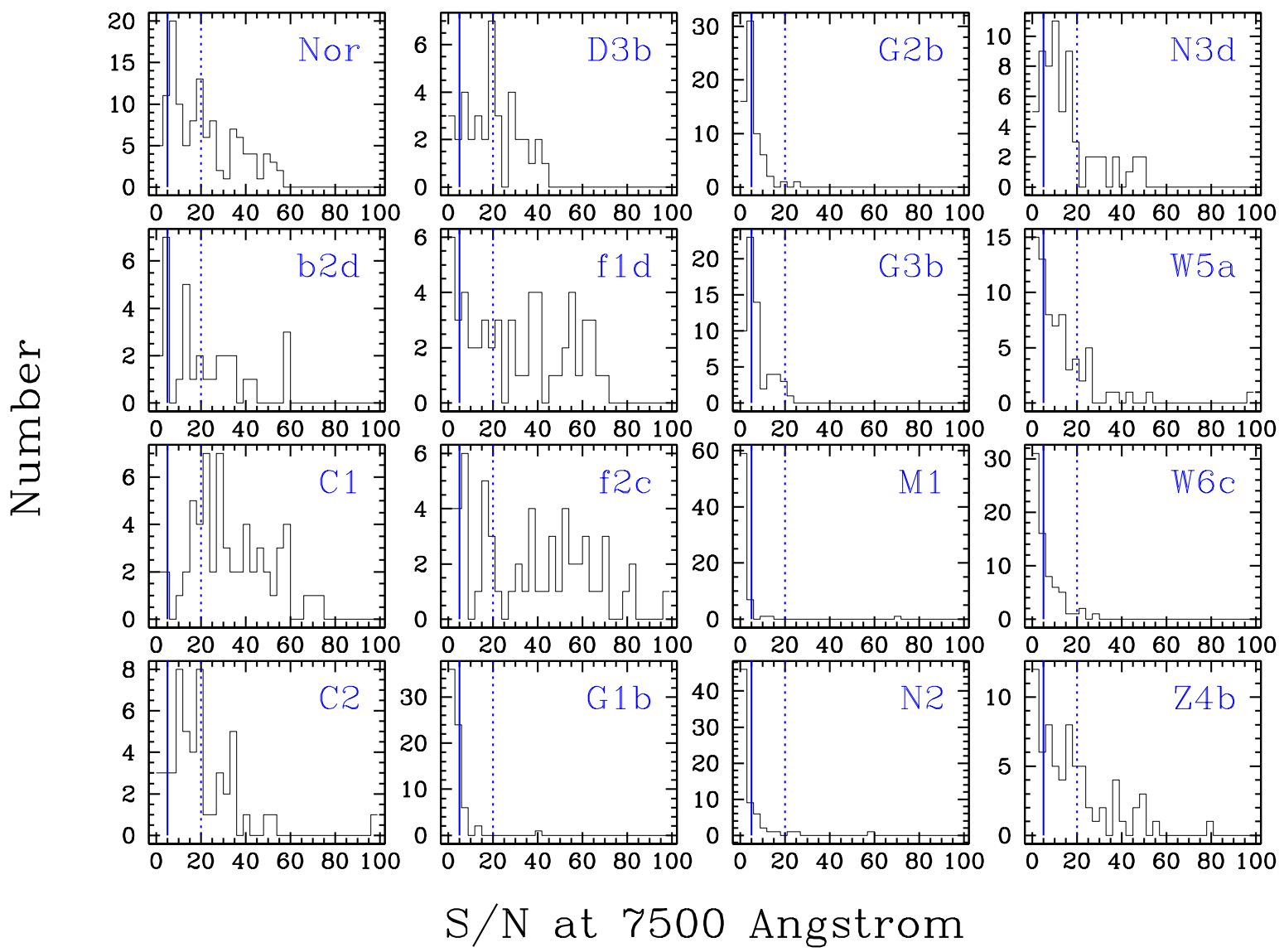

Fig. 1.- Histograms of the achieved signal-to-noise ratio in individual fiber configurations, with panel labels corresponding to rows in Table $1 . \mathrm{S} / \mathrm{N}$ is measured simply as the mean count level divided by the root-mean-square of the count level in the $7500 \AA$ continuum region of each final one-dimensional spectrum. Vertical lines drawn at $\mathrm{S} / \mathrm{N}$ values of 5 (solid) and 20 (dotted) are to guide the eye in comparing the panels. The fiber data sets have varying levels of overall quality, mostly due to weather issues. 

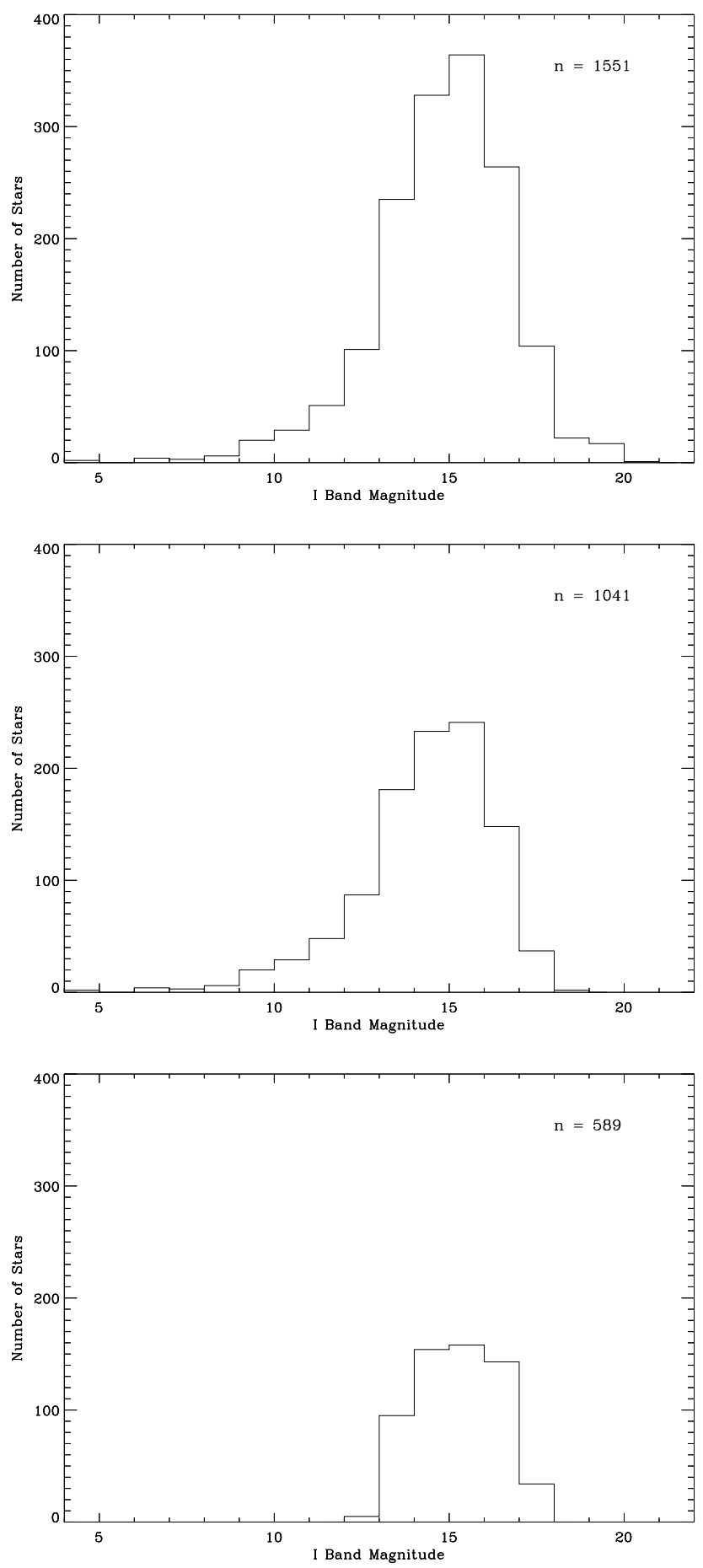

Fig. 2.- Histograms of I-band magnitudes for the H97 sample, with the top panel showing the full photometric sample, the middle panel highlighting those stars with spectral types available at the time of that publication, and the bottom panel showing those stars with spectral types (excluding limits) presented in the last column of Table 2 . There is overlap between middle and bottom panels. 


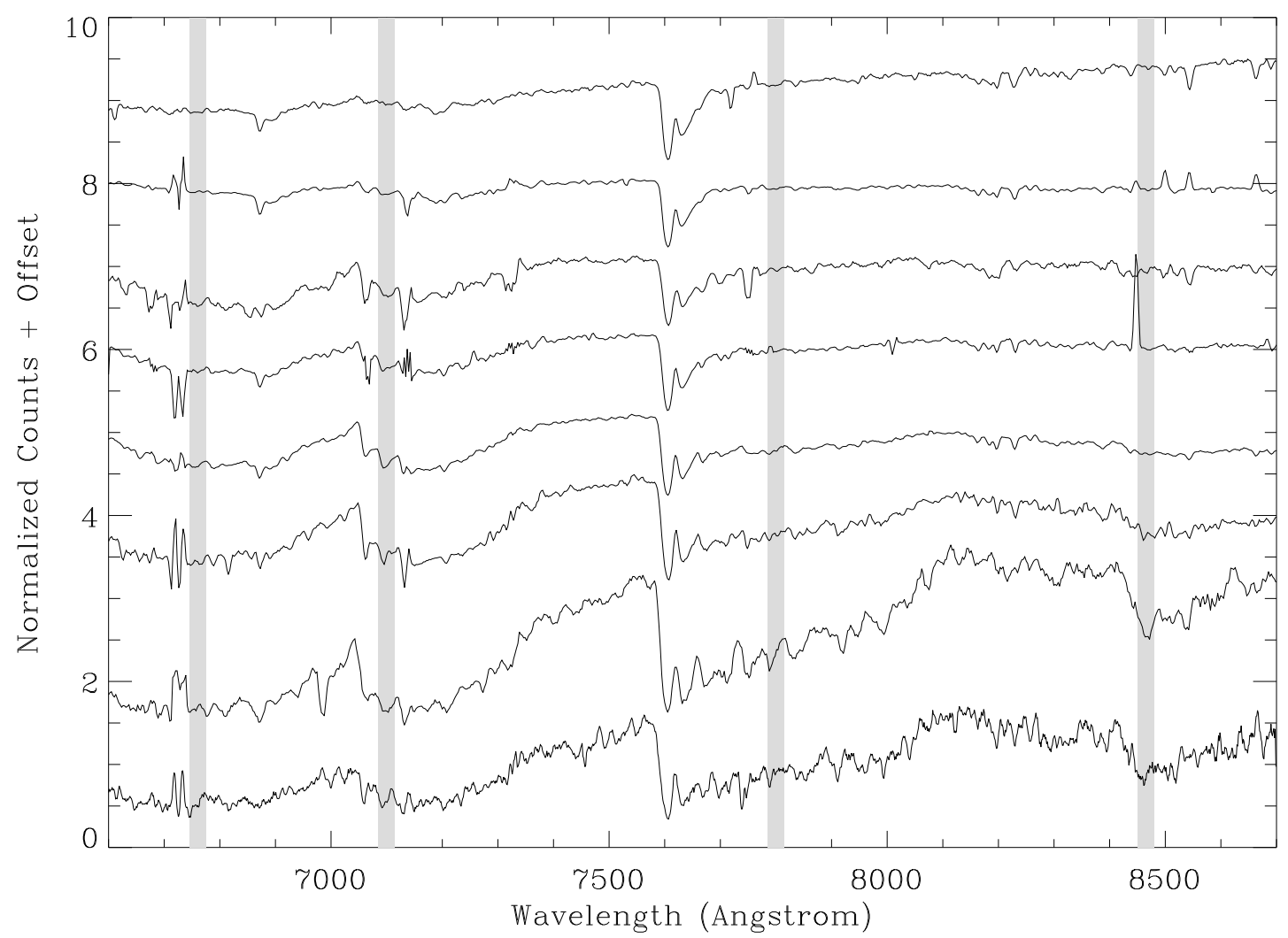

Fig. 3.- Sequence of spectra compared to the $30 \AA$-wide regions (shaded) used for the TiO band depth measurements in the spectral typing analysis. These are among the higher signal-to-noise ratio spectra in our sample, taken mostly from the WIYN/HYDRA "f2c" configuration. The objects and their spectral types are (top to bottom): H97 5170 (K5-K6), JW 762 (K8-M0e), JW 340 (M2), JW 901 (M2.5-M3.5), H97 5084 (M4), JW 812 (M5), H97 3123 (M6), and JW 1036 (M8-M9). Note the over- and/or under-subtraction in various spectra of numerous nebular emission lines, notably $\mathrm{H} \alpha$. The second spectrum from the top exhibits weak Ca II triplet emission $(\lambda \lambda 8498 \AA, 8542 \AA$, and $8662 \AA)$ as well as $\mathrm{O}$ I emission $(\lambda 8446 \AA)$, that is intrinsic to the source and not nebular contamination. The top spectrum has the Ca II lines in absorption. Note that continuum shapes can be affected by blueing due to accretion processes and/or reddening due to circumstellar and/or interstellar extinction. 

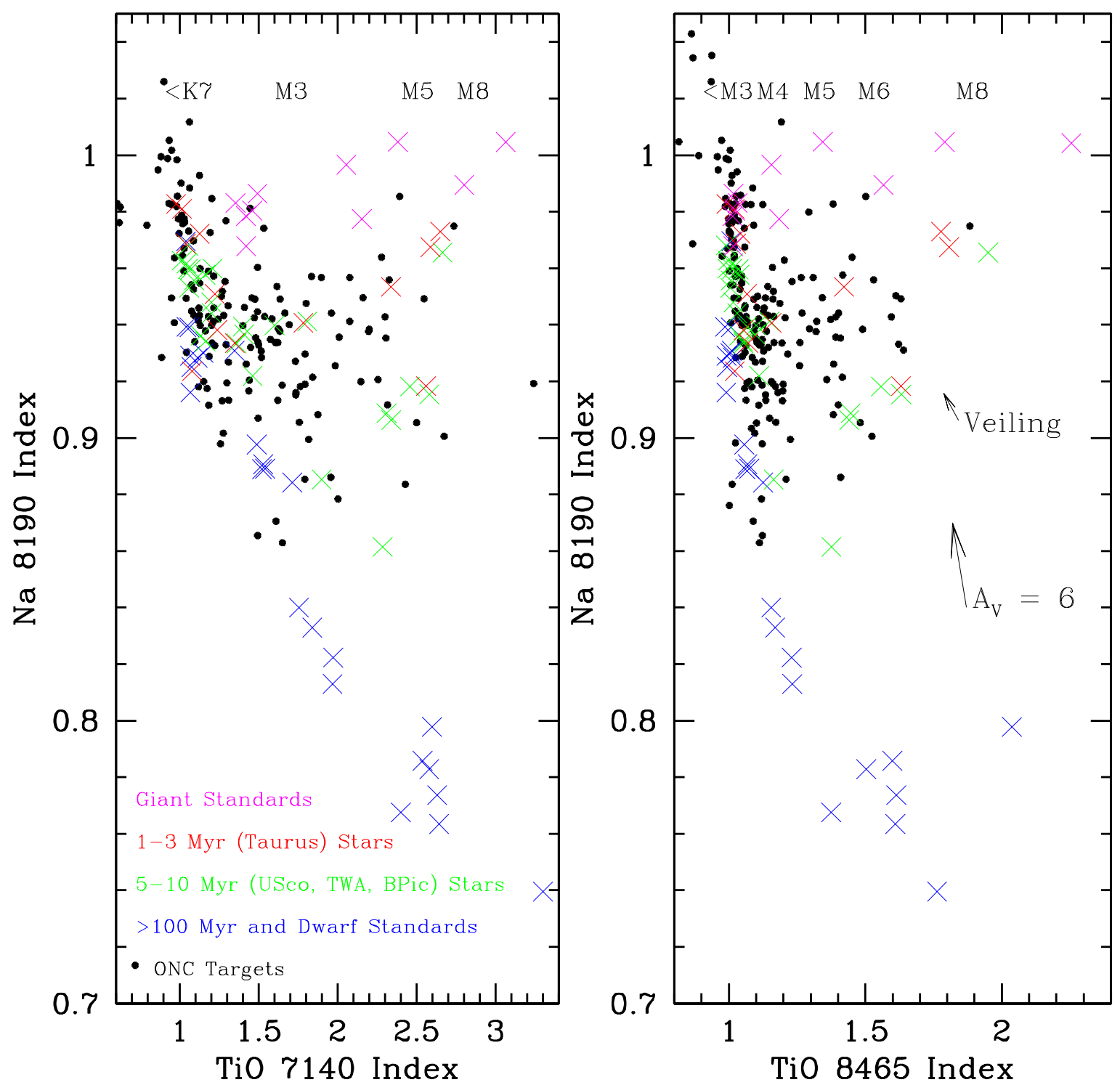

Fig. 4. - The gravity sensitive Na I $8190 \AA$ doublet spectral index is plotted as a function of the temperature sensitive TiO $7140 \AA$ index (left panel) and TiO $8465 \AA$ index (right panel), following Slesnick et al. (2006ab). ONC data (black points) can be compared to standards of known surface gravity including giants (magenta crosses), dwarfs in the field, Hyades, Pleiades, and AB Dor groups (blue crosses), and intermediate-gravity pre-main sequence stars in nearby young associations 1$10 \mathrm{Myr}$ old (red and green crosses), as indicated in the figure legend. A signal-to-noise cut has been applied to the entire ONC spectral data set with only those objects in the best $20 \%$ of our spectral data shown. In the right panel, the Slesnick et al. (2006b) vectors indicating the effects of veiling and reddening are shown. For the left panel the veiling vector would be significantly flatter (Slesnick et al. 2006a) and the reddening vector would be only slightly flatter. 


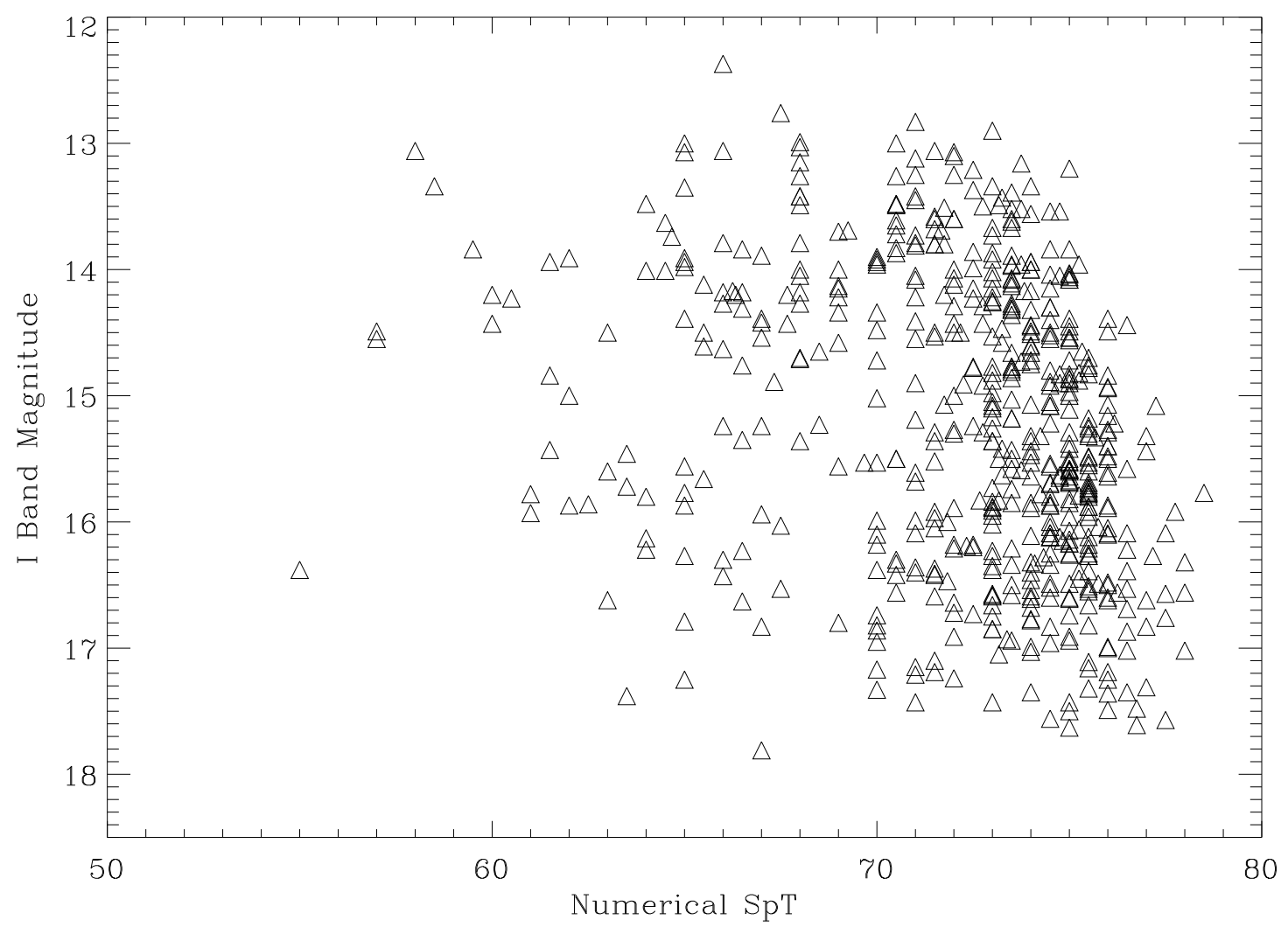

Fig. 5.- I-band magnitude from H97 vs spectral type from Table 2. Along the abscissa, spectral type (SpT) is represented numerically in a scheme where 50 corresponds to G0, 60 to K0, and 70 to M0. There is a spread in the apparent magnitudes of $\sim 5^{m}$ that is roughly constant with spectral type. We attribute the scatter largely to differential reddening, though variations among stars of the same spectral type in the amount of accretion-related continuum excess emission or in intrinsic radii are also possible contributors. 


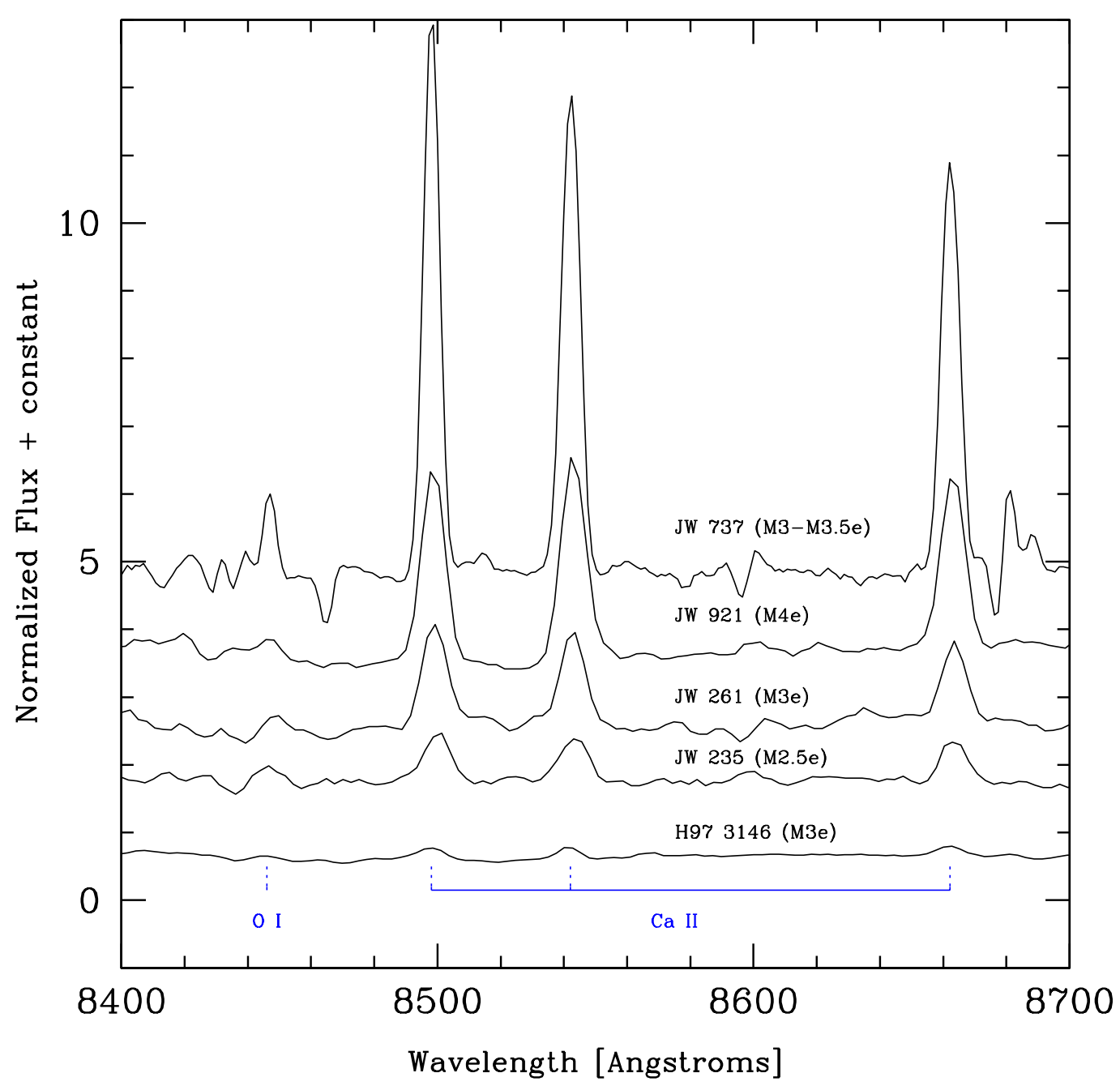

Fig. 6. - Example Ca II triplet emission spectra. The top spectrum is one of the strongest emission line sources in the sample presented here, with $\lambda 8542$ equivalent width of almost $-40 \AA$, while the bottom spectrum is one of the weaker emitters at $-1.2 \AA$. Note the variation among sources in the triplet line ratios; for classical $\mathrm{T}$ Tauri stars in Taurus, the $\lambda 8542$ line is generally the strongest with typical values of $1.20 \pm 0.19$ for the $8542 \AA$ to $8498 \AA$ ratio and $1.28 \pm 0.13$ for the $8542 \AA$ to $8662 \AA$ ratio (based on analysis of our own collection of optical spectra for representative samples). Most sources show weak O I emission along with the stronger Ca II. 


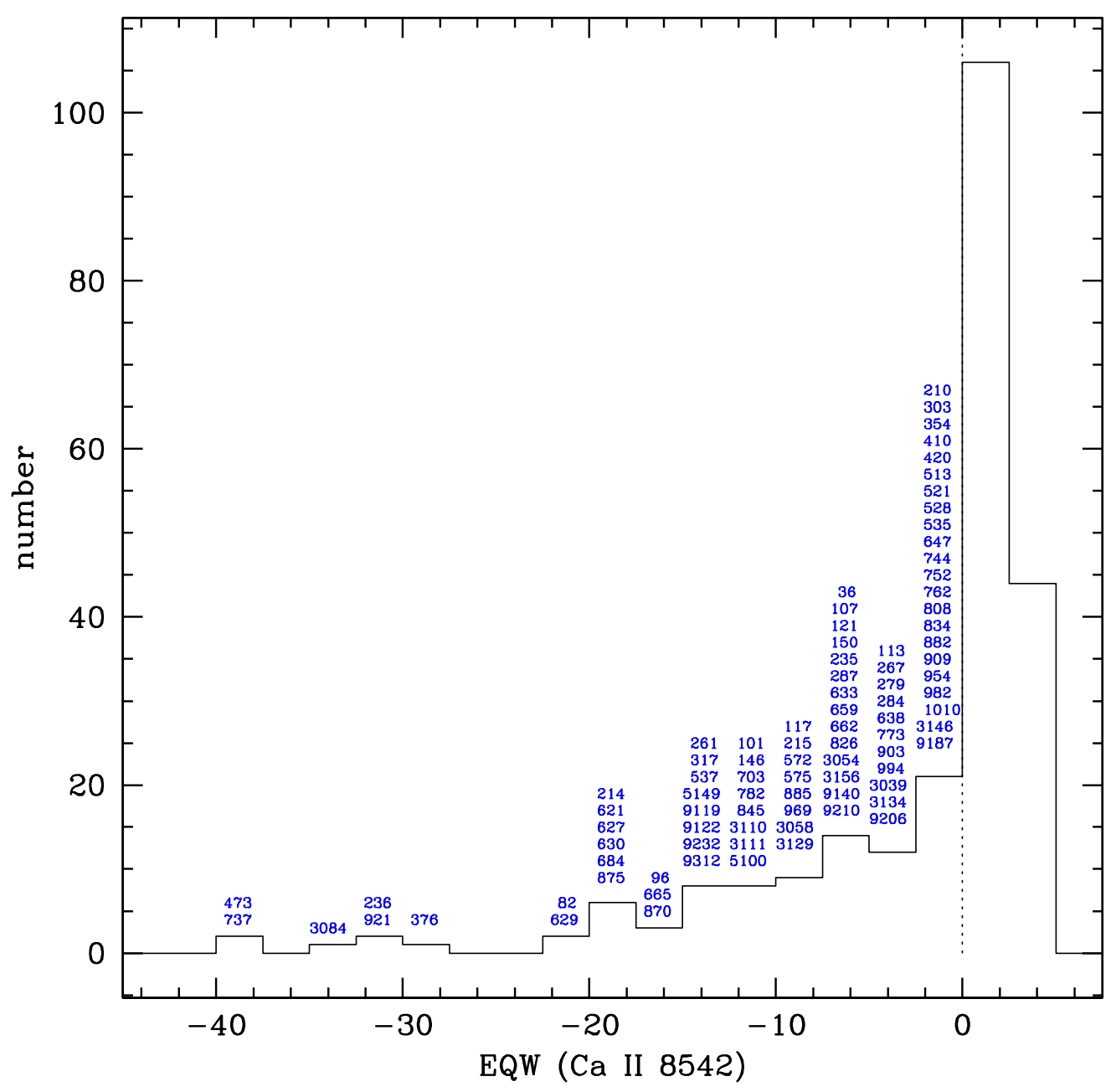

Fig. 7.- Histogram of Ca II $\lambda 8542$ equivalent widths (EQW) measured for several hundred of our spectra with adequate signal-to-noise in the continuum. Labels mark the identifiers from Table 2 of the readily diagnosed Ca II triplet emitters; each star appears only once, with equivalent widths measure from different spectra of the same object averaged. Approximately $\sim 40 \%$ of the measured spectra and $\sim 20 \%$ of the total sample have notable emission. Some stars with multiple spectra exhibited line variability up to the $25 \%$ level, which could move them between bins on this plot. 


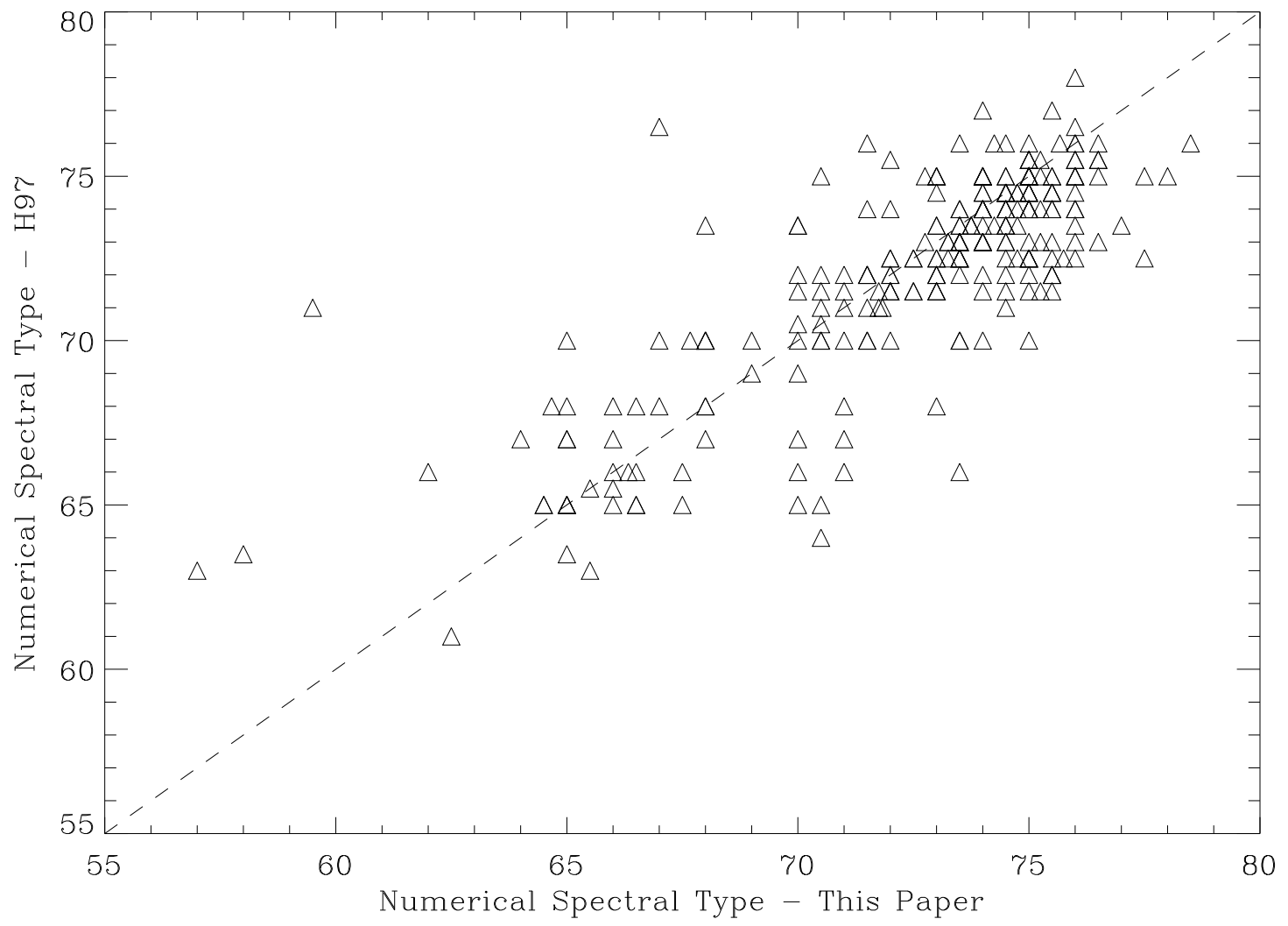

Fig. 8.- Comparison of spectral types quoted in H97 with those quoted in the last column of Table 2, derived in this study. Along both axes, spectral type is represented numerically in a scheme where 50 corresponds to G0, 60 to K0, and 70 to M0. The dashed line represents one-toone correspondence with the root-mean-squared deviation 2.25 spectral sub-classes. The largest discrepancies can be attributed to obvious errors in sky subtraction in one or the other set of spectra; this effect probably contributes to the lower level scatter as well. 

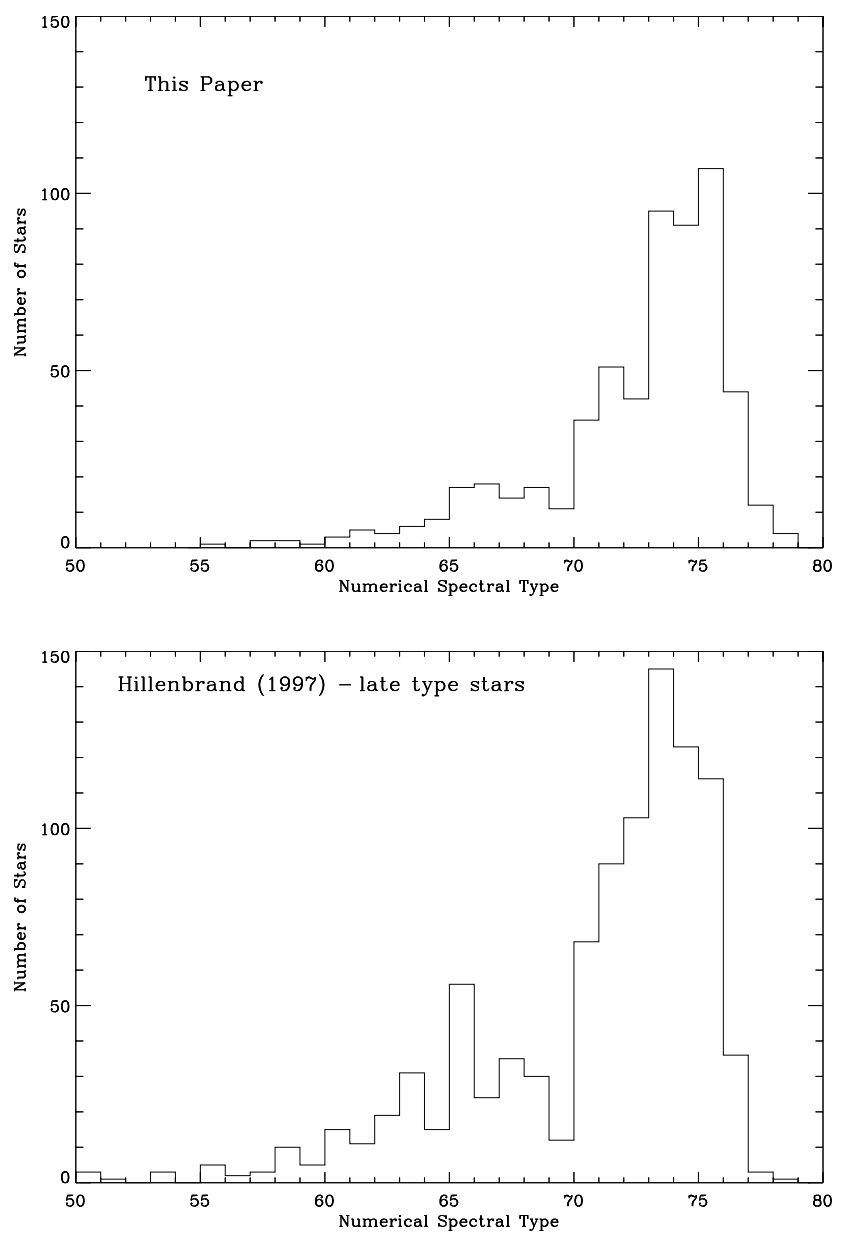

Fig. 9.- Histograms comparing the samples of newly derived spectral types and those previously reported in H97. Along the abscissa, spectral type is represented numerically in a scheme where 50 corresponds to G0, 60 to K0, and 70 to M0. 


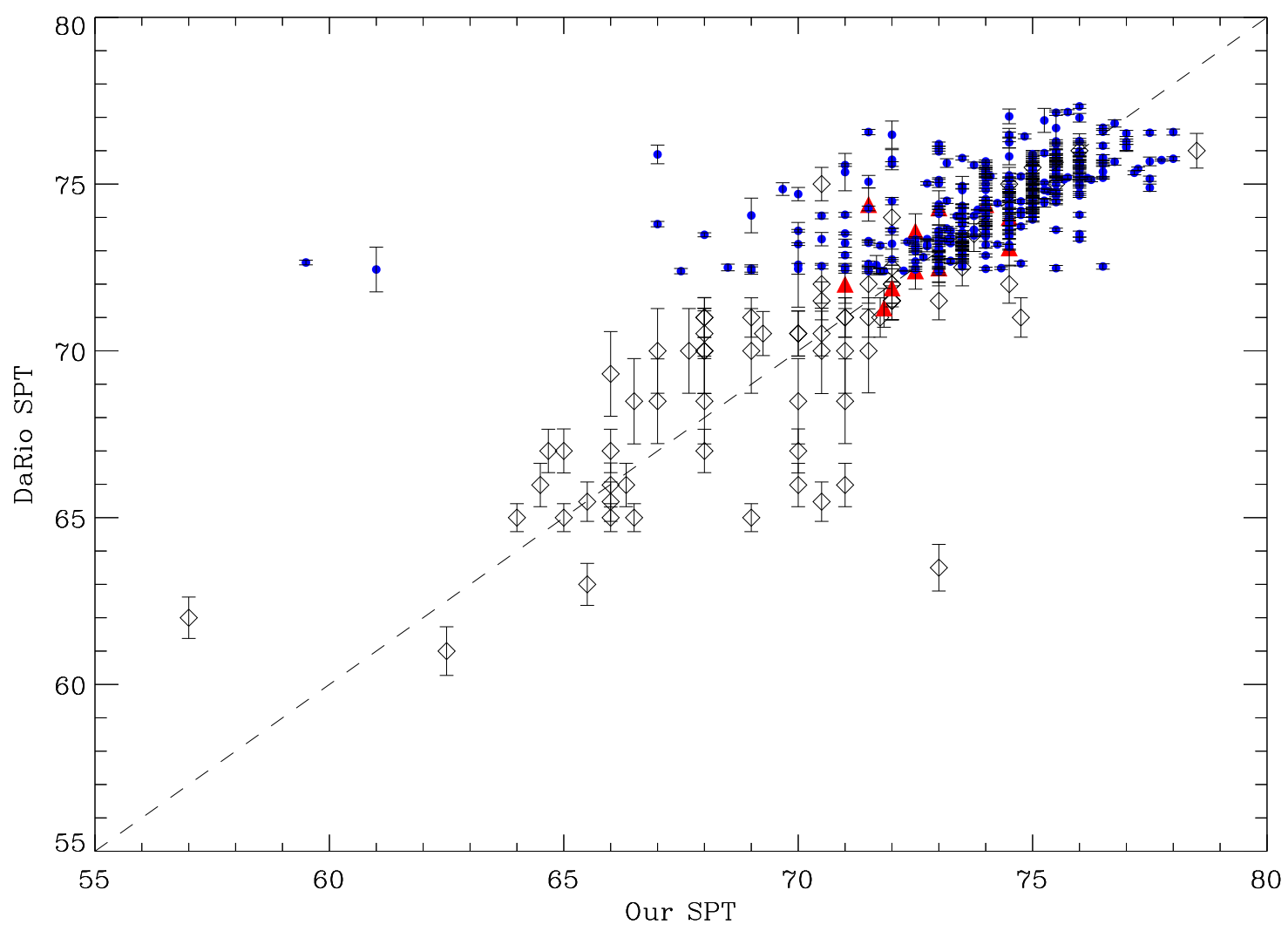

Fig. 10. - Comparison of spectral types (SpT) quoted in da Rio et al. (2012) with the ones quoted in the last column of Table 2, derived in this study. Along both axes, spectral type is represented numerically in a scheme where 50 corresponds to G0, 60 to K0, and 70 to M0. Filled circles represent spectral types based on the $7700 \AA \mathrm{TiO}$ index developed by da Rio et al. (2012). Filled triangles are sources without $7700 \AA$ types but instead have their spectral types derived from a similar 6200 $\AA \mathrm{TiO}$ index in da Rio et al. (2010). Open diamonds do not have photometric TiO types available and so the comparison for these generally earlier type stars is to the spectroscopically determined spectral type quoted in H97, which was adopted by da Rio et al. (2012). The dashed line represents one-to-one correspondence with the root-mean-squared deviation 1.75 spectral sub-classes. 


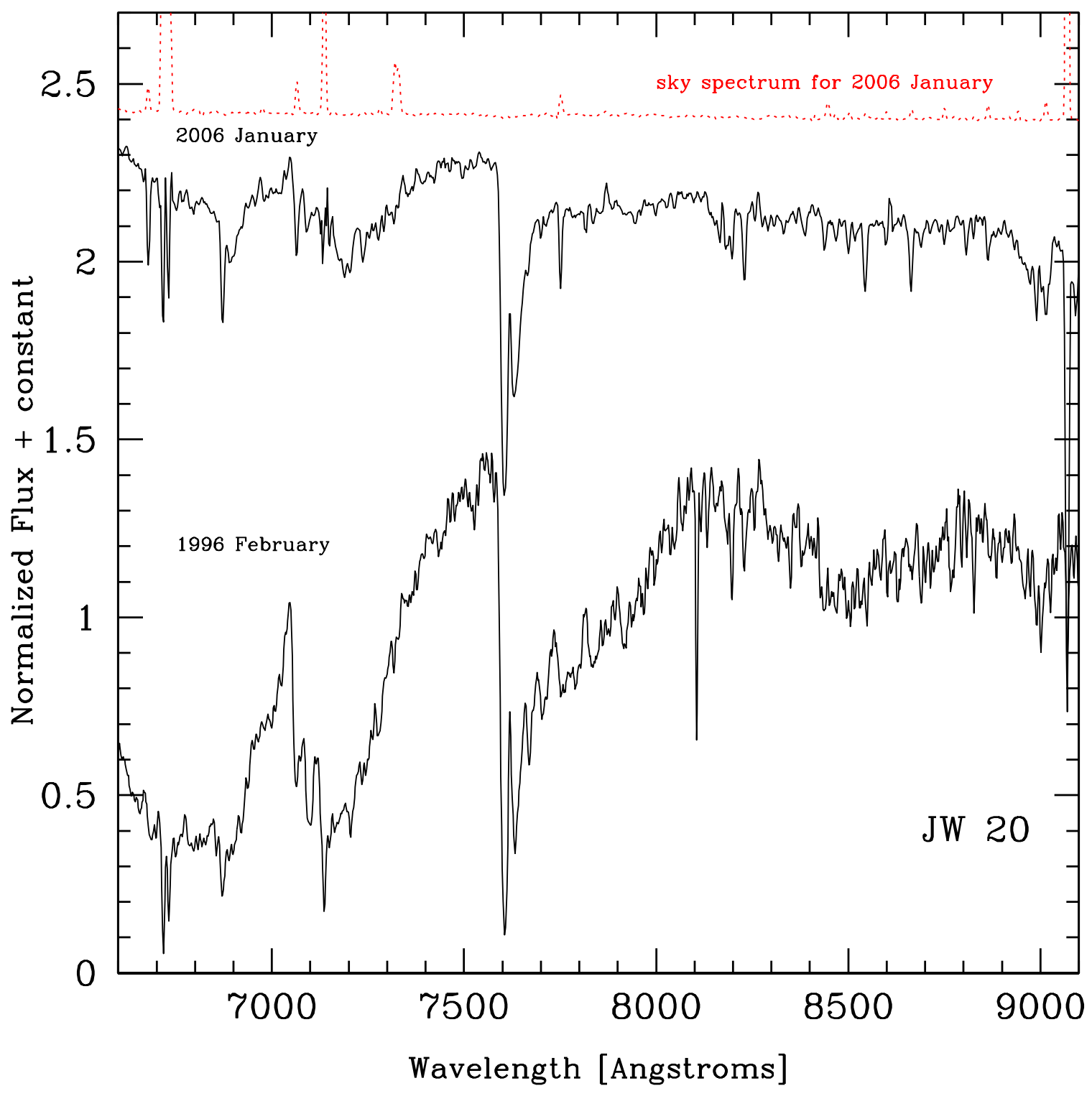

Fig. 11. - Example of an object, JW 20, whose spectrum appears to have varied significantly between observations taken about ten years apart. There is no apparent instrumental or observational reason for the discrepancy and an astrophysical origin is suggested. Furthermore, while a large change in accretion-induced veiling could change the continuum and weaken the TiO absorption, it is hard to explain the concomitant appearance of Ca II triplet absorption. A scaled down sky spectrum is also shown, to illustrate the spectral regions where nebular line contamination are most prominent. 
Table 1. Observation Log

\begin{tabular}{|c|c|c|c|c|c|c|c|}
\hline $\begin{array}{c}\text { Configuration } \\
\text { Name }\end{array}$ & Date & Instr. $^{\mathrm{a} b}$ & $\begin{array}{l}\text { On-Targets } \\
\mathrm{T}_{\text {exp. }}(\mathrm{sec})\end{array}$ & $\begin{array}{l}\text { Offset Sky } \\
\mathrm{T}_{\text {exp. }}(\mathrm{sec})\end{array}$ & $\begin{array}{c}\text { \# Target } \\
\text { Fibers }^{\mathrm{c}}\end{array}$ & $\begin{array}{l}\text { \# Sky } \\
\text { Fibers }^{\mathrm{c}}\end{array}$ & Comments \\
\hline Nor & 5 Sep. 1999 & Norris & 2700 & 900 & 120 & 13 & \\
\hline f1d & 13 Jan. 2006 & Hydra & 5400 & $2700^{\mathrm{d}}$ & 55 & 31 & thick clouds \\
\hline $\mathrm{f} 2 \mathrm{c}$ & 13 Jan. 2006 & Hydra & 5400 & $2700^{\mathrm{d}}$ & 60 & 29 & thick clouds \\
\hline $\mathrm{b} 2 \mathrm{~d}$ & 15 Jan. 2006 & Hydra & 7200 & $2400^{\mathrm{d}}$ & 30 & 54 & thick clouds \\
\hline $\mathrm{C} 1$ & 5 Jan. 2007 & Hydra & 1440 & 418 & 61 & 28 & \\
\hline $\mathrm{C} 2$ & 6 Jan. 2007 & Hydra & 1200 & 600 & 50 & 36 & \\
\hline M1 & 6 Jan. 2007 & Hydra & 2400 & 900 & 69 & 20 & poor seeing, quality \\
\hline $\mathrm{N} 2$ & 6 Jan. 2007 & Hydra & 2200 & 750 & 68 & 20 & poor seeing, quality \\
\hline G1b & 6 Jan. 2007 & Hydra & 3600 & $2100^{\mathrm{d}}$ & 69 & 19 & poor seeing, quality \\
\hline $\mathrm{G} 2 \mathrm{~b}$ & 6 Jan. 2007 & Hydra & 4800 & $1915^{\mathrm{d}}$ & 67 & 21 & \\
\hline D3b & 7 Jan. 2007 & Hydra & 1000 & 500 & 38 & 46 & \\
\hline N3d & 7 Jan. 2007 & Hydra & 2000 & 700 & 63 & 27 & \\
\hline G3b & 7 Jan. 2007 & Hydra & 4500 & $1500^{\mathrm{d}}$ & 61 & 25 & \\
\hline $\mathrm{Z} 4 \mathrm{~b}$ & 7 Jan. 2007 & Hydra & 2000 & 700 & 70 & 20 & \\
\hline W5a & 7 Jan. 2007 & Hydra & 1800 & 600 & 70 & 20 & \\
\hline W6c & 7 Jan. 2007 & Hydra & 1100 & 600 & 71 & 19 & \\
\hline
\end{tabular}

${ }^{\text {a }}$ The Norris Spectrograph was used in a setting covering $\lambda 6100-8750 \AA$ at $\mathrm{R} \sim 2000$.

${ }^{\mathrm{b}}$ The Hydra Spectrograph was used in a setting covering $\lambda$ 5000-10000 $\AA$ at $\mathrm{R} \sim 1500$.

${ }^{\mathrm{c}}$ Number of fibers assigned to target / sky positions.

${ }^{\mathrm{d}}$ Multiple exposures were combined resulting in the total exposure time. 
Table 2. ONC Spectral Types - ABBREVIATED VERSION; SEE JOURNAL FOR FULL ELECTRONIC TABLE

\begin{tabular}{|c|c|c|c|c|}
\hline Identifier ${ }^{a}$ & Right Ascension ${ }^{\mathrm{b}}$ & Declination ${ }^{b}$ & Previously Reported SpT ${ }^{c}$ & Newly Reported SpT \\
\hline 3132 & $5: 34: 11.52$ & $-5: 30: 19.7$ & . & M5.5 \\
\hline 3157 & $5: 34: 11.70$ & $-5: 33: 55.9$ & $\mathrm{M} 1(\mathrm{H})$ & \\
\hline 3126 & $5: 34: 12.91$ & $-5: 28: 48.1$ & $\mathrm{~K} 7(\mathrm{H})$ & . \\
\hline 3153 & $5: 34: 13.07$ & $-5: 33: 48.3$ & $\mathrm{M} 3(\mathrm{H})$ & $\ldots$ \\
\hline 3156 & $5: 34: 13.22$ & $-5: 33: 53.5$ & M2.5e(Sta) & $\mathrm{M} 1.5, \mathrm{M} 2 \mathrm{e}$ \\
\hline 3035 & $5: 34: 13.70$ & $-5: 17: 43.8$ & $\mathrm{G}:(\mathrm{H})$ & K4-M1 \\
\hline 3118 & $5: 34: 14.49$ & $-5: 28: 16.6$ & $\mathrm{G} 6(\mathrm{H}) \mathrm{K} 0(\mathrm{WSH})$ & \\
\hline 3007 & $5: 34: 14.90$ & $-5: 14: 18.0$ & $\mathrm{M} 2.5(\mathrm{H})$ & M3.5 \\
\hline 3024 & $5: 34: 14.97$ & $-5: 15: 49.3$ & $\mathrm{M} 1(\mathrm{H})$ & . \\
\hline 3078 & $5: 34: 15.11$ & $-5: 23: 00.0$ & $\ldots$ & M2.5 \\
\hline
\end{tabular}

${ }^{\text {a }}$ The star numbers are those listed from Jones \& Walker (1988) between \#1-1053, Parenago (1954) between \#10542999, Prosser et al. (1994) for sources given as 9000+ the Prosser number, and Hillenbrand (1997) for sources given as $3000,5000,6000$ series numbers.

${ }^{\mathrm{b}} \mathrm{J} 2000$.

${ }^{\mathrm{c}}$ The sources of the literature spectral types (SpT) are as listed in Hillenbrand (1997) with more recent additions as detailed in the text. The codes are as follows:

$\mathrm{B}=$ Blanco, 1963

CK $=$ Cohen \& Kuhi, 1979

$\mathrm{C}=$ Correia et al., 2013

$\mathrm{D}=$ Duncan, 1993

Dae $=$ Daemgen et al., 2012

$\mathrm{E}=$ Edwards et al., 1993

GS $=$ Greenstein \& Struve, 1946

$\mathrm{H}=$ Hillenbrand, 1997 and subsequent updates to electronically available table. An " $e$ " indicates emission in the CaII triplet lines. A " $<$ " indicates spectral type is earlier than that listed while ">" indicates spectral type is later than that listed.

Ham = C. Hamilton, 1994 unpublished masters thesis

Her = Herbig, quoted in Walker, 1969

$\mathrm{HP}=$ Herbig, private communication, 1996

HT $=$ Herbig \& Terndrup, 1986 or reference therein

$\mathrm{J}=$ Johnson, 1965

LA = Levato \& Abt, 1976 or Abt \& Levato, 1977

LDW = Lallemand, Duchesne, \& Walker, 1960

LR = Luhman, Rieke, et al., 2000

Luc01 = Lucas et al., 2001 (by matching given positions to known objects of similar magnitude within 2" and translating quoted $\log -\mathrm{g}=4$ Teff values into a SpT using the same Teff-SpT calibration employed in H97, largely from $\mathrm{CK}$ )

Luc06 = Lucas et al., 2006

$\mathrm{M}=$ McNamara, 1976 
$\mathrm{P}=$ Prosser \& Stauffer, private communication, 1995

Par = Parenago, 1954

Petal = Penston, Hunter, \& O'Neill, 1975 or Penston, 1973

$\mathrm{R}=$ Rhode, Herbst, Mathieu 2001 identification of SB2's

$\mathrm{RRL}=$ Riddick, Roche, Lucas 2007 (by matching given positions to known objects of similar magnitude within 2") $\mathrm{S}=$ Strand, 1958 reference (mostly to Sharpless)

Sam = A.E. Samuel, 1993 unpublished PhD thesis

$\mathrm{SBB}=$ Smith, Beckers, \& Barden, 1983

SHC $=$ Slesnick, Hillenbrand, \& Carpenter, 2004. SHC indiates optical spectral types and SHC-ir infrared spectral types.

Sta $=$ K. Stassun, private communication, 2005, February; low dispersion spectra

Ste $=$ H.C. Stempels, private communication, 2009, March; high dispersion spectra

$\mathrm{T}=$ Trumpler, 1931

vA $=$ van Altena et al., 1988

$\mathrm{W}=$ Walker, 1983

WLR = Weights et al., 2009 (by matching given positions to known objects of similar magnitude within 2"; method is similar to Luc and latest types may be too late)

WSH $=$ Wolff, Strom, \& Hillenbrand, 2004

${ }^{\mathrm{d}}$ Spectral type derived in the present study, from the Kitt Peak / WIYN data and/or the Palomar / Norris data. Many stars were classified based on several different spectra and in the cases of disagreements multiple types are listed.

e Several optically identified objects from previous studies apparently are plate defects (JW 459, JW 699) or nebular knots (PSH 9081, PSH 9326, H97 3071, H97 3089) rather than true stellar point sources. We list them here for completeness but these sources should be removed from future list of ONC stellar objects.

${ }^{\mathrm{f}}$ These sources have nebular contamination in our spectra but may include some Ca II emission contribution. 
Table 3. Additional ONC Spectral Types: Stars Fainter than those in Table 2

\begin{tabular}{|c|c|c|c|}
\hline Identifier $^{\mathrm{a}}$ & Right Ascension ${ }^{\mathrm{b}}$ & Declination ${ }^{\mathrm{b}}$ & Previously Reported SpT ${ }^{\mathrm{c}}$ \\
\hline [H97b] 10306 & $05: 34: 55.90$ & $-05: 21: 08.5$ & M6.25(RRL) \\
\hline [H97b] 10313 & 05:34:56.95 & $-05: 21: 21.9$ & M6.75(RRL) \\
\hline [H97b] 20349 & 05:35:00.90 & $-05: 21: 07.3$ & M8(WLR) \\
\hline [OW94] 013-306 & 05:35:01.3 & $-05: 23: 06$ & M7(Luc01)M9(WLR) \\
\hline [H97b] 10343 & $05: 35: 01.37$ & $-05: 24: 13.3$ & M6(Luc01)M6.5(RRL)M7.5(WLR) \\
\hline [OW94] 016-319 & 05:35:01.6 & $-05: 23: 19$ & L2.5(Luc01) > M9.5(WLR) \\
\hline [OW94] 016-430 & 05:35:01.6 & $-05: 24: 30$ & M9(Luc06)M9(WLR) \\
\hline [H97b] 10348 & 05:35:01.87 & $-05: 23: 53.7$ & M5(Luc01)M5.75(RRL)M6.5(WLR) \\
\hline [H97b] 20339 & 05:35:01.80 & $-05: 21: 06.7$ & K2(Luc01) \\
\hline [H97b] 10353 & 05:35:02.31 & $-05: 21: 23.4$ & $>$ M9.5(WLR) \\
\hline [OW94] 031-524 & 05:35:03.1 & $-05: 25: 24$ & M7.5(RRL)M8(WLR) \\
\hline 2MASS J05350313-0525364 & 05:35:03.13 & $-05: 25: 36.5$ & M8.75(RRL)>M9.5(WLR) \\
\hline [H97b] 10364 & 05:35:04.19 & $-05: 20: 12.0$ & M6.5(Luc01)M7.75(RRL)M8(WLR) \\
\hline [H97b] 20296 & 05:35:04.44 & $-05: 22: 19.5$ & late-M(RRL) \\
\hline 2MASS J05350445-0525264 & 05:35:04.46 & $-05: 25: 26.5$ & M8.5(WLR) \\
\hline [H97b] 20282 & 05:35:04.62 & $-05: 22: 44.8$ & late-M(RRL) \\
\hline 2MASS J05350467-0525508 & 05:35:04.68 & $-05: 25: 50.8$ & M8.5(RRL) \\
\hline [H97b] 20302 & 05:35:04.95 & $-05: 21: 42.8$ & mid-M(RRL) \\
\hline 2MASS J05350557-0521407 & $05: 35: 05.57$ & $-05: 21: 40.7$ & $>$ M9.5(WLR) \\
\hline [OW94] 057-247 & 05:35:05.7 & $-05: 22: 47$ & $>\mathrm{M} 9(\mathrm{Luc06})>\mathrm{M} 9.5(\mathrm{WLR})$ \\
\hline [OW94] 061-401 & 05:35:06.10 & $-05: 24: 00.6$ & M8(Luc01)>M9.5(WLR) \\
\hline [HC2000] 509 & $05: 35: 06.35$ & $-05: 22: 11.6$ & M2-M5(SHC)M2-M7(SHC-ir) \\
\hline [OW94] 066-433 & 05:35:06.6 & $-05: 24: 33$ & K4.5(Luc01) \\
\hline [H97b] 20270 & 05:35:07.06 & $-05: 25: 00.9$ & M7-M9(SHC) \\
\hline [H97b] 10380 & $05: 35: 07.23$ & $-05: 26: 38.6$ & M6.5(RRL) \\
\hline [OW94] 073-205 & 05:35:07.3 & $-05: 22: 05$ & M6(Luc01) \\
\hline [HC2000] 743 & 05:35:08.10 & $-05: 23: 15.2$ & M6:(SHC-ir) \\
\hline [HC2000] 433 & 05:35:08.11 & $-05: 22: 37.5$ & M8(SHC-ir) \\
\hline [HC2000] 400 & 05:35:08.22 & $-05: 22: 53.2$ & M7-M8(SHC)M9(SHC-ir)M8.5(RRL) \\
\hline [HC2000] 725 & $05: 35: 08.27$ & $-05: 23: 07.8$ & M7:(SHC-ir) \\
\hline [H97b] 20335 & 05:35:08.31 & $-05: 19: 37.2$ & M7.25(Luc01)M9(WLR) \\
\hline [OW94] 084-104 & 05:35:08.32 & $-05: 21: 02.4$ & L0(Luc01)>M9.5(WLR) \\
\hline [HC2000] 749 & 05:35:08.34 & $-05: 23: 21.9$ & M8(SHC-ir) \\
\hline [H97b] 20208 & 05:35:08.44 & $-05: 23: 04.9$ & M7(RRL) \\
\hline [H97b] 10391 & 05:35:08.42 & $-05: 22: 30.3$ & M2(SHC-ir) \\
\hline [OW94] 086-324 & 05:35:08.62 & $-05: 23: 24.4$ & M5.5:(SHC-ir) \\
\hline 2MASS J05350865-0520223 & 05:35:08.66 & $-05: 20: 22.4$ & M9.5(WLR) \\
\hline [HC2000] 455 & 05:35:08.93 & $-05: 22: 30.0$ & M2-M6(SHC-ir) \\
\hline [HC2000] 724 & 05:35:09.03 & $-05: 23: 26.3$ & M6:(SHC-ir) \\
\hline [OW94] 092-532 & 05:35:09.2 & $-05: 25: 32$ & M7.5(Luc06)M7.5(WLR) \\
\hline
\end{tabular}


Table 3-Continued

\begin{tabular}{|c|c|c|c|}
\hline Identifier $^{a}$ & Right Ascension ${ }^{b}$ & Declination $^{\mathrm{b}}$ & Previously Reported SpT ${ }^{\text {c }}$ \\
\hline [H97b] 20298 & 05:35:09.20 & $-05: 26: 05.5$ & M8(RRL) \\
\hline [H97b] 10403 & 05:35:09.57 & $-05: 19: 42.7$ & M6.5(Luc01)M6.75(RRL)M9(WLR) \\
\hline$[\mathrm{HC} 2000] 722$ & 05:35:09.79 & $-05: 24: 06.7$ & $\mathrm{M} 6.5(\mathrm{SHC})$ \\
\hline [H97b] 20184 & 05:35:09.91 & $-05: 24: 10.5$ & M3(SHC) \\
\hline$[\mathrm{HC} 2000] 62$ & $05: 35: 10.03$ & $-05: 25: 01.5$ & M9(SHC-ir) \\
\hline [HC2000] 90 & $05: 35: 10.38$ & $-05: 24: 51.6$ & M7.5(SHC-ir) \\
\hline [OW94] 107-453 & $05: 35: 10.7$ & $-05: 24: 53$ & M8(Luc06)M8(WLR) \\
\hline [HC2000] 529 & $05: 35: 10.88$ & $-05: 22: 06.0$ & M8(SHC-ir) \\
\hline [HC2000] 111 & $05: 35: 11.15$ & $-05: 24: 36.5$ & M9(SHC-ir) \\
\hline [HC2000] 434 & $05: 35: 11.20$ & $-05: 22: 37.8$ & M2(SHC-ir) \\
\hline [HC2000] 515 & $05: 35: 11.21$ & $-05: 22: 10.8$ & M7:(SHC-ir) \\
\hline [HC2000] 127 & $05: 35: 11.33$ & $-05: 24: 26.6$ & M0(SHC-ir) \\
\hline [HC2000] 559 & $05: 35: 11.37$ & $-05: 21: 54.0$ & M8(SHC-ir) \\
\hline [HC2000] 709 & $05: 35: 11.63$ & $-05: 22: 46.1$ & M5(SHC-ir) \\
\hline [H97b] 10420 & $05: 35: 11.67$ & $-05: 26: 08.6$ & early-M(RRL) \\
\hline [HC2000] 708 & 05:35:11.92 & $-05: 22: 50.9$ & M4(SHC-ir) \\
\hline [H97b] 20182 & $05: 35: 12.93$ & $-05: 24: 57.6$ & M5.25(RRL) \\
\hline [OW94] 137-532 & $05: 35: 13.7$ & $-05: 25: 32$ & $>\mathrm{M} 9(\mathrm{Luc06})>\mathrm{M} 9.5(\mathrm{WLR})$ \\
\hline$[\mathrm{HC} 2000] 721$ & $05: 35: 13.18$ & $-05: 24: 24.9$ & M3.5:(SHC-ir) \\
\hline [H97b] 20377 & $05: 35: 14.76$ & $-05: 28: 31.8$ & M8.5(RRL) \\
\hline [OW94] 152-717 & $05: 35: 15.2$ & $-05: 27: 17$ & $>\mathrm{M} 9(\mathrm{Luc06})>\mathrm{M} 9.5(\mathrm{WLR})$ \\
\hline$[\mathrm{HC} 2000] 600$ & $05: 35: 15.41$ & $-05: 21: 39.5$ & M5(SHC-ir) \\
\hline$[\mathrm{HC} 2000] 4$ & $05: 35: 15.56$ & $-05: 25: 46.8$ & M4.5(SHC)M5.5(SHC-ir) \\
\hline [HC2000] 565 & $05: 35: 16.01$ & $-05: 21: 53.1$ & M8(SHC-ir) \\
\hline [H97b] 20295 & $05: 35: 16.52$ & $-05: 26: 34.4$ & M8.75(RRL) \\
\hline [HC2000] 237 & $05: 35: 17.41$ & $-05: 23: 41.8$ & M2e(SHC) \\
\hline [HC2000] 469 & $05: 35: 17.58$ & $-05: 22: 27.8$ & M(SHC-ir) \\
\hline [HC2000] 162 & $05: 35: 17.58$ & $-05: 24: 09.0$ & M5.5(SHC-ir) \\
\hline [HC2000] 383 & $05: 35: 17.84$ & $-05: 22: 58.2$ & M4(SHC-ir) \\
\hline [HC2000] 764 & $05: 35: 17.97$ & $-05: 23: 53.6$ & M7.5(SHC-ir) \\
\hline [HC2000] 594 & $05: 35: 18.05$ & $-05: 21: 41.2$ & M7.5(SHC-ir) \\
\hline$[\mathrm{HC} 2000] 372$ & $05: 35: 18.08$ & $-05: 23: 01.8$ & M9(SHC-ir) \\
\hline [OW94] 183-729 & $05: 35: 18.3$ & $-05: 27: 29$ & $>\mathrm{M} 9(\mathrm{Luc06}) \mathrm{M} 8.75(\mathrm{RRL})>\mathrm{M} 9.5(\mathrm{WLR})$ \\
\hline [OW94] 183-419 & $05: 35: 18.32$ & $-05: 24: 19.9$ & M2.5(SHC-ir) \\
\hline 2MASS J05351862-0526313 & $05: 35: 18.63$ & $-05: 26: 31.4$ & M8(RRL)M7(WLR) \\
\hline [OW94] 188-658 & $05: 35: 18.8$ & $-05: 26: 58$ & $>\mathrm{M} 9($ Luc06) $>$ M9.5(WLR $)$ \\
\hline [HC2000] 409 & $05: 35: 19.04$ & $-05: 22: 50.7$ & M0(SHC) \\
\hline [HC2000] 728 & $05: 35: 19.51$ & $-05: 23: 39.7$ & M5.5(SHC-ir) \\
\hline [HC2000] 123 & $05: 35: 19.64$ & $-05: 24: 31.6$ & M0-M5(SHC)M7.5(SHC-ir) \\
\hline$[\mathrm{HC} 2000] 59$ & $05: 35: 19.68$ & $-05: 25: 05.2$ & K8-M3(SHC) \\
\hline
\end{tabular}


Table 3-Continued

\begin{tabular}{|c|c|c|c|}
\hline Identifier ${ }^{a}$ & Right Ascension ${ }^{\mathrm{b}}$ & Declination $^{\mathrm{b}}$ & Previously Reported $\mathrm{SpT}^{\mathrm{c}}$ \\
\hline [OW94] 196-659 & $05: 35: 19.6$ & $-05: 27: 00$ & mid-M(RRL) >M9.5(WLR $)$ \\
\hline [HC2000] 366 & $05: 35: 19.63$ & $-05: 23: 03.6$ & M7.5(SHC-ir) \\
\hline [HC2000] 210 & $05: 35: 19.86$ & $-05: 23: 51.6$ & >M6:e(SHC)M7(SHC-ir) \\
\hline [HC2000] 365 & $05: 35: 20.13$ & $-05: 23: 04.5$ & M7:(SHC-ir) \\
\hline [HC2000] 429 & $05: 35: 20.64$ & $-05: 22: 41.2$ & M7-M9(SHC)M7.5(SHC-ir) \\
\hline [HC2000] 732 & $05: 35: 20.77$ & $-05: 22: 39.5$ & M2.5:(SHC-ir) \\
\hline [HC2000] 731 & $05: 35: 20.79$ & $-05: 22: 36.3$ & K7:(SHC-ir) \\
\hline [H97b] 20241 & 05:35:20.90 & $-05: 25: 34.5$ & M8(SHC-ir) \\
\hline [HC2000] 403 & $05: 35: 21.02$ & $-05: 22: 54.3$ & M7(SHC-ir) \\
\hline [HC2000] 729 & $05: 35: 21.12$ & $-05: 22: 50.2$ & M7(SHC-ir) \\
\hline [HC2000] 27 & $05: 35: 21.29$ & $-05: 25: 33.2$ & M5(SHC-ir) \\
\hline [H97b] 20248 & $05: 35: 21.35$ & $-05: 25: 35.0$ & M8(SHC-ir) \\
\hline [HC2000] 15 & $05: 35: 21.61$ & $-05: 25: 40.6$ & M0-M1(SHC)M3.5(SHC-ir) \\
\hline [H97b] 10597 & $05: 35: 21.69$ & $-05: 26: 52.6$ & M7.75(RRL) \\
\hline [HC2000] 730 & $05: 35: 21.71$ & $-05: 22: 38.3$ & M4(SHC-ir) \\
\hline [HC2000] 30 & $05: 35: 21.83$ & $-05: 25: 28.4$ & M0-M3e(SHC)M2(SHC-ir) \\
\hline [H97b] 10605 & 05:35:21.99 & $-05: 24: 53.3$ & $<\mathrm{K} 7$ (SHC-ir) \\
\hline [HC2000] 55 & $05: 35: 22.12$ & $-05: 25: 07.6$ & M8(SHC-ir) \\
\hline [H97b] 10606 & $05: 35: 22.17$ & $-05: 27: 44.7$ & M5.75(RRL) \\
\hline [HC2000] 200 & $05: 35: 22.54$ & $-05: 23: 54.8$ & M3(SHC-ir) \\
\hline [HC2000] 316 & $05: 35: 23.34$ & $-05: 23: 20.8$ & M3.5-M5e(SHC) \\
\hline [HC2000] 212 & $05: 35: 23.54$ & $-05: 23: 51.0$ & M9(SHC-ir) \\
\hline [H97b] 10626 & $05: 35: 25.03$ & $-05: 24: 38.4$ & $\mathrm{M} 7(\mathrm{SHC})$ \\
\hline [HC2000] 167 & $05: 35: 25.27$ & $-05: 24: 06.5$ & M6-M8(SHC)M7.5(SHC-ir) \\
\hline [HC2000] 48 & $05: 35: 25.54$ & $-05: 25: 11.7$ & M4(SHC-ir)M6.5(WLR) \\
\hline [HC2000] 568 & $05: 35: 25.56$ & $-05: 21: 54.0$ & $<\mathrm{K} 7$ (SHC-ir) \\
\hline$[\mathrm{HC} 2000] 70$ & $05: 35: 25.67$ & $-05: 25: 02.6$ & M9(SHC-ir) \\
\hline
\end{tabular}

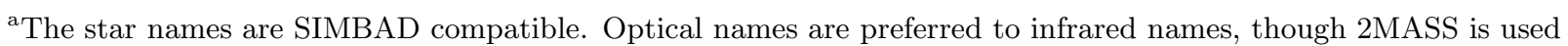
above coordinate-based optical names. It should be noted that the [H97b] identifiers in SIMBAD that begin at 10000 actually originate with Herbst et al. (2002) and those beginning with 20000 originate in Rodriguez-Ledesma et al. (2009).

${ }^{\mathrm{b}} \mathrm{J} 2000$. Note that the reported coordinates have varying levels of precision and hence accuracy in this crowded field.

${ }^{\mathrm{c}}$ The sources of the literature spectral types (SpT) have the same codes as in Table 2 . 